\title{
ABSTRACT EVOLUTION EQUATIONS $\left({ }^{1}\right)$
}

\author{
BY \\ JEROME A. GOLDSTEIN( $\left.{ }^{2}\right)$
}

1. Introduction. Let $\mathfrak{X}$ be a Banach space, and let $\mathscr{A}$ be the collection of all infinitesimal generators of strongly continuous contraction semigroups of bounded linear operators on $\mathfrak{X}$. For $A \in \mathscr{A}, \mathscr{D}(A)$ denotes the domain of $A$ and $\{T(t ; A)$, $t \geqq 0\}$ denotes the semigroup generated by $A$. Cauchy problems for abstract evolution equations of the form

$$
d u / d t=A(t) u, \quad(t \geqq s), \quad u(s)=f
$$

are considered in this paper.

In case $A(t)(\equiv A)$ is independent of $t$, then $u(\cdot)$ defined by $u(t)=T(t-s ; A) f$ is the unique strongly continuous solution of (1.1) as long as $f$ belongs to $\mathscr{D}(A)$.

More generally, if $A(t)$ depends on $t$ in a suitably "smooth" manner, then equation (1.1) can be solved for an appropriate choice of the initial data $f$. The solution will be of the form $u(t)=U(t, s) f . U(t, s)$ is a bounded linear operator on $\mathfrak{X}$,

$$
U(t, s) U(s, r)=U(t, r), \quad U(t, t)=I
$$

for $0 \leqq r \leqq s \leqq t$, and $U(\cdot, \cdot)$ is strongly continuous. The operator-valued function $U(\cdot, \cdot)$ is called an evolution operator or a generalized semigroup; the latter term will be used throughout the paper.

We now state two of the main results of the paper.

Theorem 1.1. Let $A(\cdot):[0, \infty) \rightarrow \mathscr{A}$. Suppose that the operators $T(s ; A(t))$ and $T(\sigma ; A(\tau))$ commute for all values of $t, s, \tau, \sigma$. Suppose further there is a dense linear manifold $\mathscr{D}$ in $\mathfrak{X}$ such that $\mathscr{D} \subset \mathscr{D}(A(t))$ for each $t \geqq 0$ and

$$
\lim _{t \rightarrow \tau} A(t) f=A(\tau) f
$$

for each $f \in \mathscr{D}$ and each $\tau \geqq 0$. Let

$$
\tilde{\mathscr{D}}=\bigcap_{t \geqq 0}\left(\left\{\lim _{\varepsilon \rightarrow 0+} \bigcap_{|t-\tau|<\varepsilon} \mathscr{D}(A(\tau))\right\} \cap\left\{g: \lim _{\tau \rightarrow t} A(\tau) g=A(t) g\right\}\right) .
$$

Presented to the Society, January 25, 1967 under the title Analogs of Kato's theory in the study of abstract evolution equations; received by the editors November 27, 1967 and, in revised form, October 1, 1968.

(1) This paper is based on Chapter II of the author's dissertation written under M. M. Rao at Carnegie Institute of Technology. The encouragement and guidance of Professors M. M. Rao and R. C. MacCamy is gratefully acknowledged. The research was carried out while the author was a NASA Trainee. The paper was prepared for publication at the Institute for Advanced Study under Air Force Office of Scientific Research grant AF-AFOSR-1071-66.

${ }^{(2)}$ The author is presently at Tulane University, New Orleans, Louisiana. 
Note that $\tilde{\mathscr{D}} \supset \mathscr{D}$. Then there exists a strongly continuous contraction generalized semigroup $U(\cdot, \cdot)$ such that

$$
\begin{aligned}
& (\partial / \partial t) U(t, s) f=A(t) U(t, s) f=U(t, s) A(t) f \\
& (\partial / \partial s) U(t, s) f=-U(t, s) A(s) f=-A(s) U(t, s) f
\end{aligned}
$$

for $f \in \tilde{\mathscr{D}}$ and $t \geqq s \geqq 0$. In particular, $u(t)=U(t, s) f$ defines the unique solution for (1.1) with $\|u(t)\| \leqq\|f\|$ if $f \in \tilde{\mathscr{D}}$.

Theorem 1.1 will be obtained as a consequence of a more general result in $\S 3$. Let $A(\cdot):[0, \infty) \rightarrow \mathscr{A}$, and consider the following conditions:

(A1) There is a dense linear manifold $\mathscr{D}$ in $\mathfrak{X}$ such that $\mathscr{D} \subset \bigcap_{t \in R^{+}} \mathscr{D}(A(t))$, and for each $t, \tau \in R^{+}, T(t ; A(\tau))$ leaves $\mathscr{D}$ invariant.

(A2) Let $f \in \mathfrak{X}, \eta>0$, and let $J$ be a compact interval in $R^{+}$; let $C(f, \eta, J)$ be the collection of all elements $g$ of $\mathfrak{X}$ of the form

$$
g=T\left(t_{1} ; A\left(\tau_{1}\right)\right) \cdots T\left(t_{m} ; A\left(\tau_{m}\right)\right) f
$$

where $m$ is a positive integer, $\tau_{1}, \ldots, \tau_{m} \in J$, and $\sum_{i=1}^{m} t_{i} \leqq \eta$. Suppose for each $f \in \mathscr{D}$, the map $A(\cdot) g: R^{+} \rightarrow \mathfrak{X}$ is (strongly) continuous, the continuity being uniform in $g \in C(f, \eta, J)$; thus, given $\varepsilon>0$ there exists a $\delta=\delta(\varepsilon, t, \eta, f, J)>0$ such that

$$
\|A(t+h) g-A(t) g\|<\varepsilon
$$

whenever $t, \quad t+h \in R^{+}, \quad|h|<\delta, \quad g \in C(f, \eta, J)$. Furthermore, $M(T, f, \eta, J)$ $=\sup \{\|A(t) g\|: g \in C(f, \eta, J), t \in[0, T], t \in J\}<\infty$ whenever $T$ is a positive real number.

ThEOREM 1.2. Let $A(\cdot):[0, \infty) \rightarrow \mathscr{A}$ and let (A1), (A2) hold. Then the generalized semigroup governing equation (1.1) exists. For $f \in \mathscr{D}$ and $t \geqq 0$,

$$
\lim _{h \rightarrow 0+} h^{-1}(U(t+h, t) f-f)=\lim _{h \rightarrow 0+} h^{-1}(U(t, t-h) f-f)=A(t) f,
$$

the latter limit existing for $t>0 . U(t, s): \mathscr{D} \rightarrow \bigcap_{\tau \geqq 0} \mathscr{D}(A(\tau))$ if $\mathfrak{X}$ is reflexive. Suppose that each $U(t, s)$ leaves $\mathscr{D}$ invariant. Then for $f \in \mathscr{D}, u(\cdot)=U(\cdot, s) f$ is the unique solution of the Cauchy problem

$$
\left(d^{+} / d t\right) u(t)=A(t) u(t), \quad u(s)=f, \quad\|u(t)\| \leqq\|f\| \quad(t \geqq s) .
$$

$\S 2$ deals with preliminaries. Commutative generalized semigroups are treated in $\S 3$. Theorem 1.2, the main result of this paper, is proved in $§ 5$. Several examples are also considered. In the last section, the results are briefly compared with those of Kato.

The writer wishes to thank the referee for his helpful comments.

2. Preliminaries. Let $\mathfrak{X}$ be a Banach space and $\mathscr{B}(\mathfrak{X})$ the Banach algebra of all bounded linear operators on $\mathfrak{X}$ to $\mathfrak{X}$. Let $D=\{(t, s): 0 \leqq s \leqq t<\infty\}$. A generalized semigroup in $\mathscr{B}(\mathfrak{X})$ is a map $U(\cdot, \cdot): D \rightarrow \mathscr{B}(\mathfrak{X})$ satisfying

$$
U(t, s) U(s, \tau)=U(t, \tau)
$$


whenever $0 \leqq \tau \leqq s \leqq t<\infty$, and

$$
U(t, t)=I
$$

for all $t \in R^{+}=[0, \infty)$, where $I$ is the identity operator on $\mathfrak{X} . U(\cdot, \cdot)$ is a strongly continuous contraction generalized semigroup if, in addition, $U(\cdot, \cdot) f$ is continuous on $D$ in the norm topology of $\mathfrak{X}$ for each $f \in \mathfrak{X}$, and $\|U(t, s)\| \leqq 1$ for each $(t, s) \in D$.

Proposition 2.1. Let $U(\cdot, \cdot)$ satisfy (2.1), (2.2). In order that

$$
\lim _{(\tau, \sigma) \rightarrow(t, s) ;(\tau, \sigma) \in D, \tau \geqq t} U(\tau, \sigma) f=U(t, s) f
$$

for all $f \in \mathfrak{X},(t, s) \in D$, it is sufficient that

(i) $\lim _{u \rightarrow v_{+}} U(u, v) f=\lim _{u \rightarrow v-} U(v, u) f=$ f for all $f \in \mathfrak{X}, v \in R^{+}$; that is, $U(\cdot, v)$, $U(v, \cdot)$ are strongly continuous at $v, v \in R^{+}$.

(ii) $\|U(\cdot, \cdot)\|$ is bounded on each compact subset of $D$.

Property (2.3) might be termed "right strong continuity" of $U(\cdot, \cdot)$ on $D$. In (2.3) and (i) the limits are to exist in the norm topology of $\mathfrak{X}$. Condition (2.3) becomes strong continuity of $U(\cdot, \cdot)$ on $D$ if the requirement $\tau \geqq t$ as $(\tau, \sigma) \rightarrow(t, s)$ is dropped. If this strengthened version of (2.3) holds, then (i) and (ii) necessarily follow, (ii) by the Banach-Steinhaus (Uniform Boundedness) Theorem. We point out in advance that all generalized semigroups considered in this paper will have the property that $\|U(t, s)\| \leqq 1$.

Proof of Proposition 2.1. Let $(t, s) \in D$ be fixed but arbitrary. Let $K=\{(\tau, \sigma) \in D$ : $\tau \leqq t+1\} . M=\sup _{(\tau, \sigma) \in K}\|U(\tau, \sigma)\|<\infty$ by (ii). Since the elements $(\tau, \sigma)$ of $D$ to be considered will approach $(t, s)$, it may be assumed that $(\tau, \sigma) \in K$.

Case 1. $t=s$. Let $f \in \mathfrak{X} .(\tau, \sigma) \in K$ is to approach $(t, t)$ with $\tau \geqq t$. Either $t \leqq \sigma$ or else $t \geqq \sigma$. In case $t \geqq \sigma$, using (2.2),

$$
\begin{aligned}
\|U(\tau, \sigma) f-f\| & \leqq\|U(\tau, t) U(t, \sigma) f-U(\tau, t) f\|+\|U(\tau, t) f-f\| \\
& \leqq\|U(\tau, t)\|\|U(t, \sigma) f-f\|+\|U(\tau, t) f-f\| \\
& \leqq M\|U(t, \sigma) f-f\|+\|U(\tau, t) f-f\| \rightarrow 0
\end{aligned}
$$

by (i) as $(\tau, \sigma) \rightarrow(t, t)$.

On the other hand, in case $t \leqq \sigma$,

$$
\begin{aligned}
\|U(\tau, \sigma) f-f\| & \leqq\|U(\tau, \sigma) f-U(\tau, t) f\|+\|U(\tau, t) f-f\| \\
& =\|U(\tau, \sigma)[f-U(\sigma, t) f]\|+\|U(\tau, t) f-f\| \\
& \leqq M\|U(\sigma, t) f-f\|+\|U(\tau, t) f-f\| \rightarrow 0
\end{aligned}
$$

as $(\tau, \sigma) \rightarrow(t, s)$ by (i).

Case 2. $t>s, \tau \geqq t$. Either $\sigma \leqq s$ or $\sigma \geqq s$. In case $\sigma \leqq s, \sigma \leqq s<t \leqq \tau$, and if $f \in \mathfrak{X}$,

$$
\begin{aligned}
\|U(\tau, \sigma) f-U(t, s) f\| & \leqq\|U(\tau, s) U(s, \sigma) f-U(\tau, s) f\|+\|U(\tau, t) U(t, s) f-U(t, s) f\| \\
& \leqq M\|U(s, \sigma) f-f\|+\|[U(\tau, t)-I] U(t, s) f\| \rightarrow 0
\end{aligned}
$$

as $(\tau, \sigma) \rightarrow(t, s)$ by (i). 
In case $\sigma \geqq s$, assume $\sigma \leqq t$ (since $\sigma$ is going to approach $s(<t)$ ). Then $s \leqq \sigma \leqq t \leqq \tau$, and for $f \in \mathfrak{X}$,

$$
\begin{aligned}
\|U(\tau, \sigma) f-U(t, s) f\| & \leqq\|U(\tau, t) U(t, \sigma) f-U(t, \sigma) f\|+\|U(t, \sigma) f-U(t, \sigma) U(\sigma, s) f\| \\
& =J_{1}+J_{2} .
\end{aligned}
$$

Now, $J_{2}=\|U(t, \sigma)[I-U(\sigma, s)] f\| \leqq M\|U(\sigma, s) f-f\| \rightarrow 0$ as $(\tau, \sigma) \rightarrow(t, s)$. Next,

$$
\begin{aligned}
J_{1} & =\|[U(\tau, t)-I] U(t, \sigma) f\| \\
& \leqq\|[U(\tau, t)-I][U(t, \sigma) f-U(t, s) f]\|+\|[U(\tau, t)-I] U(t, s) f\| \\
& \leqq(M+1)\|U(t, \sigma)[I-U(\sigma, s)] f\|+\|[U(\tau, t)-I] U(t, s) f\| \\
& \leqq M(M+1)\|U(\sigma, s) f-f\|+\|[U(\tau, t)-I] U(t, s) f\| \rightarrow 0
\end{aligned}
$$

as $(\tau, \sigma) \rightarrow(t, s)$. The proposition is thus proved.

COROLlaRY 2.2. Let the hypotheses of Proposition 2.1 hold, and suppose further that

$$
U(\tau, \sigma) U(t, s)=U(t, s) U(\tau, \sigma)
$$

for all $(t, s),(\tau, \sigma) \in D$. Then $U(\cdot, \cdot)$ is strongly continuous on $D$.

Proof. This follows easily by similar reasoning.

Let $\mathscr{A}$ be the collection of all infinitesimal generators of strongly continuous contraction semigroups in $\mathscr{B}(\mathfrak{X})$. For $A \in \mathscr{A}$ the semigroup generated by $A$ will be denoted by $\left\{T(s ; A), s \in R^{+}\right\}$or by $\left\{\exp (s A), s \in R^{+}\right\}$. Let $0 \leqq a<b<\infty$. Let $\Delta: a=t_{0}<t_{1}<\cdots<t_{n}=b$ be a partition of $[a, b]$; choose $\tau_{i} \in\left[t_{i-1}, t_{i}\right], i=1, \ldots, n$. Let

$$
|\Delta|=\max _{1 \leqq i \leqq n} \Delta_{i} t=\max _{1 \leqq i \leqq n}\left(t_{i}-t_{i-1}\right)
$$

be the norm of the partition. Let $A(\cdot): R^{+} \rightarrow \mathscr{A}$, and let

$$
R_{\Delta}(b, a)=T\left(\Delta_{n} t ; A\left(\tau_{n}\right)\right) \cdots T\left(\Delta_{1} t ; A\left(\tau_{1}\right)\right) .
$$

Suppose for the moment that $A(\cdot): R^{+} \rightarrow \mathscr{B}(\mathfrak{X})$ is continuous in the uniform operator topology and that $\left\{A(t): t \in R^{+}\right\}$is a commutative family of operators. Then

$$
U(t, s) f=\exp \left(\int_{s}^{t} A(u) d u\right), \quad(t, s) \in D
$$

defines a generalized semigroup in $\mathscr{B}(\mathfrak{X})$. In general, consider the following formal calculation:

$$
\begin{aligned}
\exp \left\{\int_{a}^{b} A(u) d u\right\} & \approx \exp \left\{\lim _{|\Delta| \rightarrow 0} \sum_{i=1}^{n} A\left(\tau_{i}\right) \Delta_{i} t\right\} \\
& \approx \lim _{|\Delta| \rightarrow 0} \exp \left\{\sum_{i=1}^{n} A\left(\tau_{i}\right) \Delta_{i} t\right\} \\
& \approx \lim _{|\Delta| \rightarrow 0} \prod_{i=1}^{n} \exp \left\{\Delta_{i} t A\left(\tau_{i}\right)\right\} \\
& \approx \lim _{|\Delta| \rightarrow 0} R_{\Delta}(b, a) .
\end{aligned}
$$

This is made precise in what follows. 
Proposition 2.3. Let $A(\cdot): R^{+} \rightarrow \mathscr{A}$ and let $R_{\Delta}(b, a)$ be as above. Suppose that $\lim _{|\Delta| \rightarrow 0} R_{\Delta}(b, a) f$ exists for all $f \in \mathfrak{X}$ and all $[a, b] \subset R^{+}$. If this limit is denoted by $U(b, a) f$, then $U(\cdot, \cdot)$ is a generalized semigroup in $\mathscr{B}(\mathfrak{X})$, and

$$
\lim _{b \rightarrow a+} U(b, a) f=\lim _{c \rightarrow a-} U(a, c) f=f
$$

for all $f \in \mathfrak{X}, a \in R^{+}$. Conditions (i), (ii) of Proposition 2.1 are valid, and if the commutativity condition

$$
T(s ; A(t)) T(\sigma ; A(\tau))=T(\sigma ; A(\tau)) T(s ; A(t))
$$

holds for all $s, t, \sigma, \tau \in R^{+}$, then $U(\cdot, \cdot)$ is a commutative strongly continuous contraction generalized semigroup in $\mathscr{B}(\mathfrak{X})$.

Definition. If $U(b, a) f=\lim _{|\Delta| \rightarrow 0} R_{\Delta} f$ exists for all $f \in \mathfrak{X}$ and $[a, b] \subset R^{+}$as in the proposition, then $A(\cdot)$ is called the infinitesimal propagator of $U(\cdot, \cdot)$.

The main problem considered in this paper is one of generation: when is the (unbounded) operator-valued function $A(\cdot)$ the infinitesimal propagator of a generalized semigroup in $\mathscr{B}(\mathfrak{X})$ ? This problem will be dealt with in the following sections.

$R_{\Delta}(b, a)$ may be termed a "Cauchy-Peano polygonal approximation" for $U(b, a)$ in analogy with the classical case [1].

Proof of Proposition 2.3 If $f \in \mathfrak{X}$ and $0 \leqq \tau \leqq s \leqq t<\infty$, using obvious notation,

$$
\begin{aligned}
U(t, s) U(s, \tau) f & =\lim _{|\Delta| \rightarrow 0} R_{\Delta}(t, s) \lim _{\left|\Delta^{\prime}\right| \rightarrow 0} R_{\Delta^{\prime}}(s, \tau) f \\
& =\lim _{|\Delta| \rightarrow 0} \lim _{\left|\Delta^{\prime}\right| \rightarrow 0} R_{\Delta}(t, s) R_{\Delta^{\prime}}(s, \tau) f \\
& =\lim _{\left|\Delta^{\prime \prime}\right| \rightarrow 0} R_{\Delta^{\prime}}(t, \tau) f=U(t, \tau) f .
\end{aligned}
$$

Here $\Delta^{\prime \prime}$ is the partition of $[t, \tau]$ obtained by adjoining $\Delta$ to $\Delta^{\prime}$, and $R_{\Delta^{\prime \prime}}(t, \tau)$ $=R_{\Delta}(t, s) R_{\Delta^{\prime}}(s, \tau)$ converges strongly to $U(t, \tau)$ as $\left|\Delta^{\prime \prime}\right|=\max \left(|\Delta|,\left|\Delta^{\prime}\right|\right) \rightarrow 0$. Hence $U(\cdot, \cdot)$ is a generalized semigroup in $\mathscr{B}(\mathfrak{X}) ; U(\cdot, \cdot)$ satisfies (2.1) and (2.2).

Condition (ii) of Proposition 2.1 holds since $\|U(b, a)\| \leqq 1$ whenever $0 \leqq a \leqq b<\infty$; this is immediate from the fact that $\left\|R_{\Delta}(b, a)\right\| \leqq 1$ which follows from $A(\cdot): R \rightarrow \mathscr{A}$. Condition (i) of Proposition 2.1, that is, equation (2.4), will next be shown to hold. Given $\varepsilon>0$ there is a $\delta=\delta(\varepsilon, f, a, b)>0$ such that if $\Delta$ is any partition of $[a, b]$ with $|\Delta|<\delta$, and if $R_{\Delta}(b, a)$ is a corresponding approximation for $U(b, a)$, then $\left\|R_{\Delta}(b, a) f-U(b, a) f\right\|<\varepsilon$. Let $0<K<\infty$; by considering $[a, b]$ such that $b \leqq K$, $\delta$ can be chosen to depend only on $\varepsilon, f, K$ independent of $a, b$. Then $b-a<\delta$ implies

$$
\|T(b-a ; A(c)) f-U(b, a) f\|<\varepsilon
$$

for each $c \in[a, b]$. Since $T(\cdot ; A(c))$ is strongly continuous and $T(0 ; A(c))=I$, taking first $c=a$ and then $c=b$ in (2.6) yields

$$
\limsup _{b \rightarrow a+}\|U(b, a) f-f\| \leqq \varepsilon, \quad \limsup _{a \rightarrow b-}\|U(b, a) f-f\| \leqq \varepsilon .
$$

Since $\varepsilon>0$ is arbitrary, (2.4) is thus proved. 
In case (2.5) holds, using obvious notation,

$$
\begin{aligned}
U(t, s) U(\tau, \sigma) & =\lim _{|\Delta| \rightarrow 0} \lim _{\left|\Delta^{\prime}\right| \rightarrow 0} R_{\Delta}(t, s) R_{\Delta^{\prime}}(\tau, \sigma) f \\
& =\lim _{|\Delta| \rightarrow 0} \lim _{\left|\Delta^{\prime}\right| \rightarrow 0} R_{\Delta^{\prime}}(\tau, \sigma) R_{\Delta}(t, s) f \quad \text { by }(2.5) \\
& =U(\tau, \sigma) U(t, s) f
\end{aligned}
$$

for all $(\tau, \sigma),(t, s) \in D, f \in \mathfrak{X}$. Also, $U(\cdot, \cdot)$ is strongly continuous in this case by Corollary 2.2 .

3. The commutative case. Let $A(\cdot): R^{+} \rightarrow \mathscr{A}$, so that for each $t>0, A(t)$ is the infinitesimal generator of a strongly continuous contraction semigroup $\{T(s ; A(t))$, $\left.s \in R^{+}\right\}$in $\mathscr{B}(\mathfrak{X})$. (By the Hille-Yosida Theorem [2], [5], [13], this holds if and only if $A(t)$ is a closed densely defined operator and $\lambda$ is in the resolvent set of $A(t)$ and $\left\|\lambda(\lambda I-A(t))^{-1}\right\| \leqq 1$ whenever $\lambda>0, t \in R^{+}$.) The domain of $A(t)$ will be denoted by $\mathscr{D}(A(t))$.

Consider the following set of conditions.

(B1) $T(t ; A(\tau)) T(s, A(\sigma))=T(s ; A(\sigma)) T(t ; A(\tau))$ for all $s, \sigma, t, \tau \in R^{+}$.

(B2) There is a dense linear manifold $\mathscr{D} \subset \mathfrak{X}$ such that $\mathscr{D} \subset \bigcap_{t \in R^{+}} \mathscr{D}(A(t))$, and for each $f \in \mathscr{D}$, the limits

$$
A(\tau-) f=\lim _{t \rightarrow \tau_{-}} A(t) f, A(\tau+) f=\lim _{t \rightarrow \tau^{+}} A(t) f
$$

exist (here $A(0-) f=A(0) f)$, and $A(\tau-) f=A(\tau+) f=A(\tau) f$ for all but a finite number of $\tau$ in any bounded subinterval of $R^{+}$.

(B2)' There is a dense linear manifold $\mathscr{D} \subset \mathfrak{X}$ such that $\mathscr{D} \subset \bigcap_{t \in R^{+}} \mathscr{D}(A(t))$, and for each $f \in \mathscr{D}$ and each $\tau \in R^{+}$,

$$
\lim _{t \rightarrow \tau} A(t) f=A(\tau) f
$$

Theorem 3.1. Let $A(\cdot): R^{+} \rightarrow \mathscr{A}$ and let (B1), (B2) hold. Then $A(\cdot)$ is the infinitesimal propagator of a strongly continuous contraction commutative generalized semigroup $U(\cdot, \cdot)$ in $\mathscr{B}(\mathfrak{X})$. Furthermore,

(i) if $0 \leqq s \leqq t<\infty$, if

$$
f \in \mathscr{D}(A(t+))=\bigcup_{n=1}^{\infty} \bigcap_{\tau \in[t, t+1 / n)} \mathscr{D}(A(\tau))
$$

and if $\lim _{\tau \rightarrow t+} A(\tau) f=A(t) f$, then

$$
\lim _{h \rightarrow 0+} h^{-1}(U(t+h, s) f-U(t, s) f)=A(t) U(t, s) f=U(t, s) A(t) f
$$

(ii) if $0 \leqq s<t<\infty$, if

$$
f \in \mathscr{D}(A(t-))=\bigcup_{n=1}^{\infty} \bigcap_{\tau \in(t-1 / n, t]} \mathscr{D}(A(\tau)),
$$

and if $\lim _{\tau \rightarrow t-} A(\tau) f=A(t) f$, then

$$
\lim _{h \rightarrow 0+}-h^{-1}(U(t-h, s) f-U(t, s) f)=A(t) U(t, s) f=U(t, s) A(t) f
$$


(iii) if $0 \leqq s<t<\infty$, if $f \in \mathscr{D}(A(s+))$, and if $\lim _{\tau \rightarrow s+} A(\tau) f=A(s) f$, then

$$
\lim _{h \rightarrow 0+} h^{-1}(U(t, s+h) f-U(t, s) f)=-U(t, s) A(s) f=-A(s) U(t, s) f
$$

(iv) if $0<s \leqq t<\infty$, if $f \in \mathscr{D}(A(s-))$, and if $\lim _{\tau \rightarrow s-} A(\tau) f=A(s) f$, then

$$
\lim _{h \rightarrow 0+}-h^{-1}(U(t, s-h) f-U(t, s) f)=-U(t, s) A(s) f=-A(s) U(t, s) f .
$$

Theorem 1.1 is an immediate consequence of Theorem 3.1, so it suffices to prove Theorem 3.1. The advantage of hypothesis (B2) is that it enables one to deal with "piecewise continuous" $A(\cdot)$; in particular, $A(\cdot)$ can be the step function

where $0=t_{0}<t_{1}<\cdots \rightarrow \infty, A_{i} \in \mathscr{A}$.

$$
A(\cdot)=\sum_{i=1}^{\infty} A_{i} \chi_{\left[t_{i} . t_{i+1}\right)}(\cdot),
$$

For the proof of Theorem 3.1 the following three lemmas will be useful.

LeMma 3.2. Let $\mathfrak{X}$ be a Banach space, and let $T_{1}, \ldots, T_{n}, S_{1}, \ldots, S_{n} \in \mathscr{B}(\mathfrak{X})$ be contractions such that $U V=V U$ for all $U, V \in\left\{T_{1}, \ldots, T_{n}, S_{1}, \ldots, S_{n}\right\}$. Then for each $f \in \mathfrak{X}$,

$$
\left\|\left(T_{1} \cdots T_{n}\right) f-\left(S_{1} \cdots S_{n}\right) f\right\| \leqq \sum_{i=1}^{n}\left\|T_{i} f-S_{i} f\right\| .
$$

This result is proved in Feller [3, p. 252]; it also follows from Lemma 5.2.

Lemma 3.3. Let $A, B \in \mathscr{A}$, and suppose that

$$
T(s ; A) T(t ; B)=T(t ; B) T(s ; A)
$$

for all $s, t \in R^{+}$. Then, if $t \in R^{+}$and $f \in \mathscr{D}(A) \cap \mathscr{D}(B)$,

$$
\|T(t ; A) f-T(t ; B) f\| \leqq t\|A f-B f\| .
$$

Proof. The result is trivial for $t=0$, so assume $t$ is positive. If $f \in \mathscr{D}(A) \cap \mathscr{D}(B)$,

$$
\begin{aligned}
\|T(t ; A) f-T(t ; B) f\| & =\left\|[T(t / n ; A)]^{n} f-[T(t / n ; B)]^{n} f\right\| \\
& \leqq n\|T(t / n ; A) f-T(t / n ; B) f\| \quad \text { by Lemma } 3.2 \\
& =n \cdot(t / n)\left\|(t / n)^{-1}(T(t / n ; A) f-f)-(t / n)^{-1}(T(t / n ; B) f-f)\right\| \\
& \rightarrow t\|A f-B f\|
\end{aligned}
$$

as $n \rightarrow \infty$. Since the left side of the above inequality does not depend on $n$, the result follows.

The author is indebted to Professor Peter D. Lax for pointing out that a standard trick gives the following simple and direct alternate proof:

$$
\begin{aligned}
\|T(t ; A) f-T(t ; B) f\| & =\left\|\int_{0}^{1} \frac{d}{d s}(T(t s ; A) T(t(1-s) ; B) f) d s\right\| \\
& =\left\|\int_{0}^{1} T(t s ; A) T(t(1-s) ; B)(t A-t B) f d s\right\| \\
& \leqq t\|A f-B f\| .
\end{aligned}
$$


Lemma 3.4. Let $\left\{T(t ; A), t \in R^{+}\right\},\left\{T(t ; B), t \in R^{+}\right\}$be strongly continuous semigroups in $\mathscr{B}(\mathfrak{X})$ such that $T(s ; A)$ and $T(t ; B)$ commute for all values of $s, t \in R^{+}$. Let $f \in \mathscr{D}(A)$. Then for each $s \in R^{+}, T(s ; B) f \in \mathscr{D}(A)$ and

$$
A T(s ; B) f=T(s ; B) A f .
$$

The obvious proof is omitted.

Proof of Theorem 3.1. Let the hypotheses of the theorem hold. Let $0 \leqq a<b<\infty$. Suppose that $\Delta^{1}, \Delta^{2}$ are two partitions of $[a, b]$; for $i=1,2$,

$$
\begin{gathered}
\Delta^{i}: a=t_{0}^{i}<t_{1}^{i}<\cdots<t_{n_{i}}^{i}=b, \quad \Delta_{j}^{i} t=t_{j}^{i}-t_{j-1}^{i}, \quad \tau_{j}^{i} \in\left[t_{j-1}^{i}, t_{j}^{i}\right], \\
\left|\Delta^{i}\right|=\max _{1 \leqq j \leqq n_{i}} \Delta_{j}^{i} t . \text { Let } f \in \mathscr{D} \text {. For } i=1,2 \text {, set } \\
R_{\Delta^{i}} f=\prod_{j=1}^{n_{i}} T\left(\Delta_{j}^{i} t ; A\left(\tau_{j}^{i}\right)\right) f .
\end{gathered}
$$

By (B1), the order in which the factors appear in the above product is irrelevant.

Let $\varepsilon>0$ be given. A $\delta=\delta(\varepsilon, f, a, b)>0$ will be determined such that $\left|\Delta^{1}\right|,\left|\Delta^{2}\right|<\delta$ implies $\left\|R_{\Delta^{1}} f-R_{\Delta^{2}} f\right\|<\varepsilon$. Then it will follow that $U(b, a) f=\lim _{|\Delta| \rightarrow 0} R_{\Delta} f$ exists.

Let $\Delta^{3}$ be the common refinement of $\Delta^{1}, \Delta^{2} ; \Delta^{3}: a=t_{0}^{3}<t_{1}^{3}<\cdots<t_{m}^{3}=b$, where $t_{0}^{3}=a$, and once $t_{j}^{3}$ has been defined, $t_{j+1}^{3}$ is the smallest of $t_{1}^{1}, \ldots, t_{n_{1}}^{1}, t_{1}^{2}, \ldots, t_{n_{2}}^{2}$ which is larger than $t_{j}^{3}$. For $i=1,2, R_{\Delta}{ }^{4} f$ can be written as

$$
R_{\Delta^{\natural}} f=\prod_{j=1}^{m} T\left(\Delta_{j}^{3} t ; A\left(s_{j}^{i}\right)\right) f,
$$

where $s_{j}^{i}$ is defined in the following manner: $s_{j}^{i}=\tau_{k}^{i}$, where $k$ is the unique subscript such that $t_{k-1}^{i} \leqq t_{j-1}^{3}<t_{j}^{3} \leqq t_{k}^{i}$. Note that the semigroup property has been used in obtaining (3.1).

Using (3.1) and (B1) one gets

$$
\begin{aligned}
\left\|R_{\Delta^{1}} f-R_{\Delta^{2}} f\right\| & =\left\|\prod_{j=1}^{m} T\left(\Delta_{j}^{3} t ; A\left(s_{j}^{1}\right)\right) f-\prod_{j=1}^{m} T\left(\Delta_{j}^{3} t ; A\left(s_{j}^{2}\right)\right) f\right\| \\
& \leqq \sum_{j=1}^{m}\left\|T\left(\Delta_{j}^{3} t ; A\left(s_{j}^{1}\right)\right) f-T\left(\Delta_{j}^{3} t ; A\left(s_{j}^{2}\right)\right) f\right\| \quad \text { by Lemma } 3.2 \\
& \leqq \sum_{j=1}^{m} \Delta_{j}^{3} t\left\|A\left(s_{j}^{1}\right) f-A\left(s_{j}^{2}\right) f\right\|
\end{aligned}
$$

by Lemma 3.3, since $f \in \mathscr{D} \subset \bigcap_{t \in R^{+}} \mathscr{D}(A(t))$. Let $\tau_{1}^{*}, \ldots, \tau_{L}^{*}$ be the points of discontinuity of $A(\cdot) f$ on $[a, b]$. By (B2), $L$ is finite, and given $\varepsilon_{1}>0$ there is a $\delta_{1}>0$ such that $\|A(s) f-A(t) f\|<\varepsilon_{1}$ whenever $s, t \in[a, b],|s-t|<\delta$, and none of $\tau_{1}^{*}, \ldots, \tau_{L}^{*}$ belongs to the closed interval between $s$ and $t$. Choose 
$\varepsilon_{1}=\varepsilon /(b-a+2 L M)$, where $M=\sup _{a \leqq t \leqq b}\|A(t) f\|<\infty$ by (B2). Let $\delta=$ $\min \left(\delta_{1} / 2, \varepsilon_{1}\right)$. Then from (3.2),

$$
\begin{aligned}
\left\|R_{\Delta^{1}} f-R_{\Delta^{2}} f\right\| & \leqq \sum_{j=1}^{m} \Delta_{j}^{3} t\left\|A\left(s_{j}^{1}\right) f-A\left(s_{j}^{2}\right) f\right\| \\
& \leqq \sum_{j=1}^{m} \Delta_{j}^{3} t \varepsilon_{1}+\Sigma^{\prime} \\
& =(b-a) \varepsilon_{1}+\Sigma^{\prime}
\end{aligned}
$$

if $\left|\Delta^{1}\right|,\left|\Delta^{2}\right|<\delta$, where $\Sigma^{\prime}$ is the sum of all terms of the form

$$
\Delta_{j}^{3} t\left\|A\left(s_{j}^{1}\right) f-A\left(s_{j}^{2}\right) f\right\|
$$

where $\left[t_{j-1}^{3}, t_{j}^{3}\right]$ contains one or more of the exceptional points $\tau_{1}^{*}, \ldots, \tau_{L}^{*}$. There are certainly no more than $2 L$ such terms, each of which is bounded by

$$
\Delta_{j}^{3} t \cdot M \leqq M \delta \leqq M \varepsilon_{1}
$$

if $\left|\Delta^{1}\right|,\left|\Delta^{2}\right|<\delta$, and in this case

$$
\begin{aligned}
\left\|R_{\Delta^{1}} f-R_{\Delta^{2}} f\right\| & <(b-a) \varepsilon_{1}+2 L M \varepsilon_{1} \quad \text { by (3.3) and the above } \\
& =\varepsilon .
\end{aligned}
$$

It follows from this and from $\left\|R_{\Delta^{\natural}}\right\| \leqq 1$ and $\overline{\mathscr{D}}=\mathfrak{X}$ that

$$
U(b, a) g=\lim _{|\Delta| \rightarrow 0} R_{\Delta} g
$$

exists for all $g \in \mathfrak{X}$, and all $[a, b] \subset R^{+}$. By Proposition 2.3, $U(\cdot, \cdot): D \rightarrow \mathscr{B}(\mathfrak{X})$ is a strongly continuous contraction commutative generalized semigroup with infinitesimal propagator $A(\cdot)$.

Now let $0 \leqq s \leqq t<\infty$ and $f \in \mathscr{D}(A(t+))$ be such that $\lim _{\tau \rightarrow t+} A(\tau) f=A(t) f$. Then, letting $g=U(t, s) f$ and using obvious notations, for $h>0$,

$$
\begin{aligned}
h^{-1}(U(t+h, s) f-U(t, s) f)= & h^{-1}(U(t+h, t) g-g) \\
= & \lim _{|\Delta| \rightarrow 0}\left[\prod_{i=1}^{n} T\left(\Delta_{i} t ; A\left(\tau_{i}\right)\right) g-\prod_{i=1}^{n} T\left(\Delta_{i} t ; A(t)\right) g\right] \\
& +h^{-1}(T(h ; A(t)) g-g) \\
= & f_{1}+f_{2} .
\end{aligned}
$$

Here the fact that

$$
T(h ; A(t))=\prod_{i=1}^{n} T\left(\Delta_{i} t ; A(t)\right)=\lim _{|\Delta| \rightarrow 0} \prod_{i=1}^{n} T\left(\Delta_{i} t ; A(t)\right)
$$

has been used.

Let $R_{\Delta}=\prod_{i=1}^{n} T\left(\Delta_{i} t ; A\left(\tau_{i}\right)\right)$ be an approximation for $U(t, s)$, and let $F \in \mathscr{D}(A(\tau))$. By repeated application of Lemma 3.4,

$$
A(\tau) R_{\Delta} F=R_{\Delta} A(\tau) F
$$


Letting $|\Delta| \rightarrow 0$ and using the fact that $A(\tau)$ is a closed operator it follows that $U(t, s) F \in \mathscr{D}(A(\tau))$, and

$$
A(\tau) U(t, s) F=U(t, s) A(\tau) F .
$$

By (3.4), with $\tau=t, F=f$, it follows that

$$
\lim _{h \rightarrow 0} f_{2}=A(t) g=A(t) U(t, s) f=U(t, s) A(t) f .
$$

It will now be shown that $\lim _{h \rightarrow 0} f_{1}=0$.

$$
\begin{aligned}
\left\|f_{1}\right\| & =\lim _{|\Delta| \rightarrow 0} h^{-1}\left\|\prod_{i=1}^{n} T\left(\Delta_{i} t ; A\left(\tau_{i}\right)\right) g-\prod_{i=1}^{n} T\left(\Delta_{i} t ; A(t)\right) g\right\| \\
& \leqq \liminf _{|\Delta| \rightarrow 0} h^{-1} \sum_{i=1}^{n} \Delta_{i} t\left\|A\left(\tau_{i}\right) g-A(t) g\right\| \quad \text { by Lemmas 3.2,3.3 } \\
& \leqq \liminf _{|\Delta| \rightarrow 0} h^{-1} \sum_{i=1}^{n} \Delta_{i} t \sup _{\tau \in[t, t+h]}\|A(\tau) g-A(t) g\| \\
& =\liminf _{|\Delta| \rightarrow 0} \sup _{\tau \in[t, t+h]}\|A(\tau) g-A(t) g\| .
\end{aligned}
$$

Using (3.4),

$$
\|A(\tau) g-A(t) g\|=\|U(t, s)(A(\tau) f-A(t) f)\| \leqq\|A(\tau) f-A(t) f\| .
$$

Therefore, since $\lim _{\tau \rightarrow t+} A(\tau) f=A(t) f$, it follows that $\lim _{h \rightarrow 0} f_{1}=0$. This completes the proof that

$$
\lim _{h \rightarrow 0+} h^{-1}(U(t+h, s) f-U(t, s) f)=A(t) U(t, s) f=U(t, s) A(t) f .
$$

The remaining three assertions of Theorem 3.1 are proved in a similar manner. The commutativity assumption (B1) and equation (3.4) are used. The proof of the theorem is thus completed.

If $u(t)=U(t, s) f, t \geqq s, f \in \mathfrak{X}$, then $\|u(\cdot)\|$ is a monotone nonincreasing function on $[s, \infty)$ since $\|U(\tau, \sigma)\| \leqq 1$ for each $(\tau, \sigma) \in D$. This is a slightly stronger form of the last assertion of Theorem 1.1.

In applying Theorems 3.1 and 1.1 to abstract evolution equations it is desirable to have a criterion to determine when the commutativity relation (B1) is fulfilled. Roughly speaking, if $\mathfrak{X}=C(\Omega)$ or $L^{p}(\Omega)$, if $\mathscr{D}_{0}\left(=C^{4}(\Omega)\right.$ or $C_{0}^{4}(\Omega)$ or $C_{0}^{\infty}(\Omega)$, etc.) is dense in $\mathfrak{X}$, and if $A, B$ are differential operators having closed extensions (denoted again by $A, B$ ) which are infinitesimal generators, then, under suitable conditions, $A B f=B A f$ for all $f \in \mathscr{D}_{0}$ implies $(\lambda I-A)^{-1}$ and $(\lambda I-B)^{-1}$ commute for large $\lambda$, which implies that $T(s ; A)$ and $T(t ; B)$ commute.

THEOREM 3.5. Let $A, B$ be infinitesimal generators of strongly continuous semigroups in $\mathscr{B}(\mathfrak{X})$. Let $\mathscr{D}_{0}$ be a linear manifold in $\mathfrak{X}$ such that $\mathscr{D}_{0} \subset \mathscr{D}(A B) \cap \mathscr{D}(B A)$ and $A B f=B A f$ for all $\mathscr{D}_{0}$. Suppose there is a $\lambda_{0}>0$ such that

$$
\mathscr{D}_{\lambda}=\left\{(\lambda I-A)(\lambda I-B) f: f \in \mathscr{D}_{0}\right\}
$$

is dense in $\mathfrak{X}$ for each $\lambda>\lambda_{0}$. Then $T(s ; A)$ commutes with $T(s ; B)$ for all $t, s \in R^{+}$. 
Proof. The proof depends on the exponential formula

$$
T(t ; A) f=\lim _{n \rightarrow \infty}\left(I-t n^{-1} A\right)^{-n} f, \quad f \in \mathfrak{X},
$$

(see [5, p. 352]). Let

$$
\mathscr{D}_{\mu \lambda}=(\mu I-A)(\lambda I-B)\left(\mathscr{D}_{0}\right)=(\mu I-A)(\lambda I-A)^{-1}\left(\mathscr{D}_{\lambda}\right) .
$$

Then for $\lambda, \mu$ sufficiently large, $\mathscr{D}_{\mu \lambda}$ is dense in $\mathfrak{X}$ since $\mathscr{D}_{\lambda}$ is and $(\mu I-A)(\lambda I-A)^{-1}$ is an automorphism of $\mathfrak{X}$. The bounded operators $(\mu I-A)^{-1}(\lambda I-B)^{-1}$ and $(\lambda I-B)^{-1}(\mu I-A)^{-1}$ clearly coincide on $\mathscr{D}_{\mu \lambda}$. Hence $(\mu I-A)^{-1}$ and $(\lambda I-B)^{-1}$ commute for $\lambda, \mu$ large enough. Hence, for $f \in \mathfrak{X}, t, s \in R^{+}$,

$$
\begin{aligned}
T(s ; A) T(t ; B) f & =\lim _{n \rightarrow \infty}\left(I-s n^{-1} A\right)^{-n} \lim _{n \rightarrow \infty}\left(I-t n^{-1} B\right)^{-n} f \\
& =\lim _{n \rightarrow \infty}\left(I-s n^{-1} A\right)^{-n}\left(I-t n^{-1} B\right)^{-n} f \\
& =\lim _{n \rightarrow \infty}\left(I-t n^{-1} B\right)^{-n}\left(I-s n^{-1} A\right)^{-n} f \\
& =T(t ; B) T(s ; A) f .
\end{aligned}
$$

Here we have used (3.5), the commutativity of $(I-u A)^{-1}$ and $(I-v B)^{-1}$ for $u$, $v$ sufficiently small, and the fact that if $S_{n}, T_{n}, S, T \in \mathscr{B}(\mathfrak{X})$ and if $S_{n} \rightarrow S, T_{n} \rightarrow T$ strongly, then $S_{n} T_{n} \rightarrow S T$ strongly.

Let $A, B$ be as in Theorem 3.5, and let $\lambda \in \rho(A) \cap \rho(B)$, where $\rho(T)$ is the resolvent set of the operator $T$. Then $(\lambda I-A)^{-1}$ commutes with $(\lambda I-B)^{-1}$ if and only if $(\lambda I-A)$ commutes with $(\lambda I-B)$ on the domain

$$
\mathscr{M}_{\lambda}=\left\{(\lambda I-A)^{-1}(\lambda I-B)^{-1} f: f \in \mathfrak{X}\right\} .
$$

COROllary 3.6. Let $A, B$ be infinitesimal generators of strongly continuous semigroups in $\mathscr{B}(\mathfrak{X})$. Let $\mathscr{M}_{\lambda}$ be defined as above, and suppose that $A B f=B A f$ for all

$$
f \in \mathscr{M}=\lim _{\mu \rightarrow \infty} \bigcup_{\lambda>\mu} \mathscr{M}_{\lambda}\left(=\bigcup_{v \in R^{+}} \bigcap_{\mu \geqq v} \bigcup_{\lambda>\mu} \mathscr{M}_{\lambda}\right) \text {. }
$$

Then the conclusion of Theorem 3.5 holds.

Proof. According to the remark preceding the statement of the corollary, $(\lambda I-A)^{-1}$ and $(\lambda I-B)^{-1}$ commute for large enough $\lambda$. The proof of Theorem 3.5 now applies.

In concluding this section the following consequence of Theorems $3.1,1.1$ can be given.

Corollary 3.7. Let $A(\cdot): R^{+} \rightarrow \mathscr{A}$ satisfy (B1) and (B2) (or (B2)'). Let a $(\cdot)$ : $R^{+} \rightarrow R^{+}$be continuous. If $B(t)=a(t) A(t)$, then $B(\cdot): R^{+} \rightarrow \mathscr{A}$, and $B(\cdot)$ satisfies (B1) and (B2) (or (B2)'). Consequently the conclusions of Theorem 3.1 (or 1.1) apply to the generalized semigroup of which $B(\cdot)$ is the infinitesimal propagator.

Proof. If $A$ is an infinitesimal generator and $a \geqq 0$, then $a A$ is an infinitesimal generator and $T(t ; a A)=T(a t ; A)$. Since $a(\cdot)$ is continuous, the corollary follows immediately. 
4. Examples. In this section Theorem 1.1 will be applied to (spatially homogeneous) Cauchy problems of the form

$$
\frac{\partial u}{\partial t}=\sum_{i, j=1}^{n} a_{i j}(t) \frac{\partial^{2} u}{\partial x_{i} \partial x_{j}}+\sum_{i=1}^{n} b_{i}(t) \frac{\partial u}{\partial x_{i}}+c(t) u, \quad u(0, x)=f(x)
$$

First consider the semigroups associated with constant coefficient parabolic equations in $R^{n}$. Define an operator $L$ formally by

$$
(L u)(x)=\sum_{i, j=1}^{n} a_{i j} \frac{\partial^{2} u}{\partial x_{i} \partial x_{j}}(x)+\sum_{i=1}^{n} b_{i} \frac{\partial u}{\partial x_{i}}(x)+c u(x)
$$

Here $x=\left(x_{1}, \ldots, x_{n}\right) \in R^{n}$. Let $\mathfrak{X}$ be the Banach space of bounded continuous functions on $R^{n}$ that vanish at $\infty ; \mathfrak{X}$ is equipped with the supremum norm. Assume that the matrix $\left(a_{i j}\right)$ is positive definite and that $c \leqq 0$. Then there exists a strongly continuous contraction semigroup $\left\{T(t), t \in R^{+}\right\}$with infinitesimal generator $A$, such that $A f=L f$ for all

$f \in C_{0}^{2}=\left\{g: g, \frac{\partial g}{\partial x_{i}}, \frac{\partial^{2} g}{\partial x_{i} \partial x_{j}} \in \mathfrak{X}, 1 \leqq i, j \leqq n\right.$, and $g$ has compact support in $\left.R^{n}\right\}$

Moreover, there exists a nonnegative (Green's) function $G(\cdot, \cdot): R^{+} \times R^{n} \rightarrow R^{+}$ such that

$$
(T(t) f)(x)=\int_{R^{n}} f(y) G(t, x-y) d y
$$

for all $f \in \mathfrak{X}$. These assertions can be proved, for example, by the parametrix method (Friedman [4]) or by Fourier transforms. As noted above, $\mathscr{D}(A) \supset C_{0}^{2}$; however, a complete characterization of $\mathscr{D}(A)$ does not seem to be known (Nelson [10]).

Proposition 4.1. Suppose that $a_{i j}(\cdot), b_{i}(\cdot), c(\cdot)(1 \leqq i, j \leqq n)$ are continuous functions on $R^{+}$to $R^{1}$, that $\left(a_{i j}(t)\right)$ is a positive definite symmetric matrix for each $t \in R^{+}$, and that $f \in C_{0}^{2}$. Then there exists a unique (classical) solution of the Cauchy problem (4.1) which, as a function of $x$ for fixed $t$, vanishes at $\infty$, and which depends continuously on the initial data.

Proof. Let the hypotheses of the theorem hold. It suffices to find the solution $u_{T}$ on $[0, T] \times R^{n}$ with $T>0$ arbitrary. (By uniqueness, $u_{T}(t, x)=u_{S}(t, x)$ whenever $0 \leqq t \leqq \min (S, T)$.) As far as finding $u_{T}$ is concerned, the values $c(t)$ for $t>T$ are irrelevant. In particular, $u_{T}(\cdot, \cdot)$ is the solution of the problem

$$
\begin{aligned}
\frac{\partial v}{\partial t} & =L(t) v \equiv \sum_{i, j=1}^{n} a_{i j}(t) \frac{\partial^{2} v}{\partial x_{i} \partial x_{j}}+\sum_{i=1}^{n} b_{i}(t) \frac{\partial v}{\partial x_{i}}+c_{T}(t) v, \\
v(0, x) & =f(x),
\end{aligned}
$$


where $c_{T}(t)=c(\min (t, T)) . c_{T}(\cdot)$ is bounded above on $R^{+}$, say by $M_{T}$. Let $v_{T}$ $=e^{-M_{T} t} u_{T}$. Then $v_{T}$ satisfies

$$
\frac{\partial v}{\partial t}=\left(-M_{T} I+L(t)\right) v, \quad v(0, x)=f(x) .
$$

More explicitly,

$$
\frac{\partial v}{\partial t}=\sum_{i, j=1}^{n} a_{i j}(t) \frac{\partial^{2} v}{\partial x_{i} \partial x_{j}}+\sum_{i=1}^{n} b_{i}(t) \frac{\partial v}{\partial x_{i}}+\left(c_{T}(t)-M_{T}\right) v
$$

and $c_{T}(t)-M_{T} \leqq 0$ for all $t \in R^{+}$. The point of this calculation is that it may be assumed, for convenience, that $c(t) \leqq 0$ for all $t \in R^{+}$.

By the remarks at the beginning of this section, the hypotheses of the theorem and the condition $c(t) \leqq 0$ imply that for each $\tau \in R^{+}$there is a strongly continuous contraction semigroup $\left\{T(t ; A(\tau)), t \in R^{+}\right\}$such that $\mathscr{D}(A(\tau)) \supset C_{0}^{2}$ and $A(\tau)=L(\tau) f$ for all $f \in C_{0}^{2}$.

It will now be shown that the rest of the conditions of Theorem 1.1 are satisfied. Take $\mathscr{D}=C_{0}^{2}$. If $f \in \mathscr{D}$,

$$
\begin{aligned}
\|A(t) f-A(\tau) f\|= & \|L(t) f-L(\tau) f\| \\
\leqq & \sum_{i, j=1}^{n}\left|a_{i j}(t)-a_{i j}(\tau)\right|\left\|\frac{\partial^{2} f}{\partial x_{i} \partial x_{j}}\right\|+\sum_{i=1}^{n}\left|b_{i}(t)-b_{i}(\tau)\right|\left\|\frac{\partial f}{\partial x_{i}}\right\| \\
& +|c(t)-c(\tau)|\|f\| \rightarrow 0
\end{aligned}
$$

as $t \rightarrow \tau$ by the continuity of the coefficients. Therefore (B2) ${ }^{\prime}$ is satisfied. To see that (B1) holds, let $G^{\tau}(\cdot, \cdot)$ be the Green's function corresponding to $\{T(t ; A(\tau))$, $\left.t \in R^{+}\right\}$. Then, using (4.2), whenever $t, \tau, s, \sigma \in R^{+}, x \in \Omega, f \in \mathfrak{X}$,

$$
\begin{aligned}
T(s ; A(\sigma)) T(t ; A(\tau)) f(x) & =\int_{R^{n}}(T(t ; A(\tau)) f)(x-z) G^{\sigma}(z, s) d z \\
& =\int_{R^{n}} \int_{R^{n}} f(x-z-y) G^{\tau}(y, t) G^{\sigma}(z, s) d y d z,
\end{aligned}
$$

and similarly

$$
T(t ; A(\tau)) T(s ; A(\sigma)) f(x)=\int_{R^{n}} \int_{R^{n}} f(x-y-z) G^{\sigma}(z, s) G^{\tau}(y, t) d z d y .
$$

The expressions in (4.3) and (4.4) are equal by Fubini's Theorem, therefore (B1) holds.

By Theorem 1.1, $A(\cdot)$ is the infinitesimal propagator of a strongly continuous contraction generalized semigroup $U(\cdot, \cdot)$ in $\mathscr{B}(\mathfrak{X})$. For $f \in \mathscr{D}=C_{0}^{2}, u(t, \cdot)=$ $(U(t, 0) f)(\cdot)$ is the unique solution of the problem

$$
\frac{\partial u}{\partial t}=\sum_{i, j=1}^{n} a_{i j}(t) \frac{\partial^{2} u}{\partial x_{i} \partial x_{j}}+\sum_{i=1}^{n} b_{i}(t) \frac{\partial u}{\partial x_{i}}+c(t) u
$$


$\lim _{t \rightarrow 0} u(t, x)=f(x)$ uniformly in $x \in R^{n}$ and $\sup _{x \in R^{n}}|u(t, x)| \leqq \sup _{x \in R^{n}}|f(x)|$ for all $t \in R^{+}$. In case $c(\cdot)$ is not necessarily nonpositive, the stability condition becomes

$$
\sup _{x \in R^{n}}|u(t, x)| \leqq \sup _{x \in R^{n}} e^{M_{t}}|f(x)|
$$

where, as before, $M_{t}=\max _{s \in[0, t]} c(s)$.

The proof of the above proposition can be modified to show that the Cauchy problem for the equations

$$
\begin{aligned}
& (\partial u / \partial t)(t, x)=(L(t) u)(t, x)+d(t) u(t, x+e(t)), \\
& (\partial u / \partial t)(t, x)=(L(t) u)(t, x)+d(t) \sum_{m=0}^{\infty} u(t, x+e(t)) / m !
\end{aligned}
$$

can be solved classically if the hypotheses of Proposition 4.1 hold and if $d(\cdot)$ : $R^{+} \rightarrow R$ and $e(\cdot): R^{+} \rightarrow R^{n}$ are continuous.

We conclude this section by solving a higher order parabolic equation with constant coefficients. Let $\Omega$ be a bounded domain in $R^{n}$ with a sufficiently smooth boundary (of class $C^{3 p-1}$ will do). If $\alpha_{1}, \ldots, \alpha_{j}$ are integers between 1 and $n$, set $\alpha=\left(\alpha_{1}, \ldots, \alpha_{j}\right),|\alpha|=j$, and

$$
D^{\alpha}=\partial^{j} / \partial x_{\alpha_{1}} \cdots \partial x_{\alpha_{j}}
$$

The formula

$$
\|f\|_{k}^{2}=\sum_{|\alpha| \leqq k} \int_{\Omega}\left|D^{\alpha} f(x)\right|^{2} d x
$$

defines an inner product norm on the space

$$
G_{k}=\left\{f \in C^{\infty}(\Omega):\|f\|_{k}<\infty\right\} .
$$

Let $H_{k}(\Omega)$ be the completion of $G_{k}$ in $\|\cdot\|_{k}$, and let $H_{k}^{0}(\Omega)$ be the completion in $\|\cdot\|_{k}$ of $C_{0}^{\infty}(\Omega)$, the $C^{\infty}$ functions with compact support in $\Omega$.

Let $A(t)$, for each fixed $t \in R^{+}$, be an elliptic operator of order $2 p$ with constant coefficients (see, for example, Friedman [4, Chapter 10]). By the results of Lax and Milgram [9], there exists a real number $\gamma(t)$ such that $A(t)-\gamma(t) I \in \mathscr{A}$ and

$$
\mathscr{D}(A(t))=\mathscr{D}(A(t)-\gamma(t) I)=H_{2 p}(\Omega) \cap H_{p}^{0}(\Omega)
$$

independent of $t$. If the coefficients of $A(t)$ depend continuously on $t$, then $\gamma(\cdot)$ can be taken to be a continuous function of $t$.

Proposition 4.2. Let $L$ be an elliptic operator of order $2 p$ with coefficients depending on $t$ but not on $x$; moreover, suppose the dependence of the coefficients on $t$ is continuous. Then the problem

$$
u_{t}=L u, \quad u(0)=f, \quad D^{\alpha} u(t)=0 \quad \text { on } \partial \Omega,|\alpha| \leqq p
$$

has a strong $\left(\right.$ i.e. $\left.L^{2}\right)$ solution $u(\cdot)$ if $f \in H_{2 p}(\Omega) \cap H_{p}^{0}(\Omega)$. $u(\cdot)$ depends on $f$ continuously (in norm), and $u(\cdot)$ satisfies the boundary conditions in the sense that

$$
u(\cdot): R^{+} \rightarrow H_{2 p}(\Omega) \cap H_{p}^{0}(\Omega) .
$$


The last statement follows from Lemma 5.2 which will be proved in the next section. The rest of the proof can be patterned after that of Proposition 4.1, except that Theorem 3.5 is used to prove the commutativity. The proof is omitted.

5. The general case. Theorem 1.2 will be proved in this section. Prior to the proof some remarks will be made concerning the hypotheses of this theorem. In the following section concrete examples are given to show that the conditions (A1), (A2) are nonvacuous; these examples help illustrate the nature of (A1), (A2).

We isolate part of the statement of Theorem 1.2 as a lemma.

Lemma 5.1. Let the hypotheses of Theorem 1.2 hold. Then for each $(t, s) \in D$,

if $\mathfrak{X}$ is reflexive.

$$
U(t, s): \mathscr{D} \rightarrow \bigcap_{\tau \in R^{+}} \mathscr{D}(A(\tau))
$$

In particular, it follows that each $U(t, s)$ leaves $\mathscr{D}$ invariant in case $\mathscr{D}=\mathscr{D}(A(t))$ is independent of $t$. The proof of Lemma 5.1 will be presented after the proof of Theorem 1.2.

Note that if the conditions of Theorem 1.1 hold, and if $\mathscr{D}=\mathscr{D}(A(t))$ is independent of $t$, then (A2) is fulfilled. This will be proved in the next paragraph. So in case $\mathscr{D}=\mathscr{D}(A(t))$ is independent of $t$, Theorem 1.2 is a generalization of Theorem 1.1 ; in case $\mathscr{D}(A(t))$ does depend on $t$, this need not be true. (In the example of the preceding section, $\mathscr{D}=C_{0}^{2}$ is not left invariant by each $T(s ; A(t))$ so that (A1) is not satisfied.)

Now suppose that $\mathscr{D}=\mathscr{D}(A(t))$ is independent of $t$ and that the hypotheses of Theorem 1.1 hold. Note that in this case $T(s ; A(t))$ leaves $\mathscr{D}=\mathscr{D}(A(t))$ invariant. If $f \in \mathscr{D}$ and $s, t, \tau \in R^{+}$,

$$
A(t) T(s ; A(\tau)) f=T(s ; A(\tau)) A(t) f
$$

by Lemma 3.4. Let $f \in \mathscr{D}, \varepsilon>0, t \in R^{+}$. Then there is a $\delta=\delta(\varepsilon, t, f)>0$ such that

$$
\|A(t+h) f-A(t) f\|<\varepsilon
$$

if $t+h \in R^{+},|h|<\delta$. If $g \in C(f, \eta, J)$ for some choice of $\eta, J$, then $g$ can be written as

$$
g=T\left(t_{1} ; A\left(\tau_{1}\right)\right) \cdots T\left(t_{n} ; A\left(\tau_{n}\right)\right) f
$$

and so if $t+h \in R^{+},|h|<\delta$,

$$
\begin{aligned}
\|A(t+h) g-A(t) g\| & =\left\|\{A(t+h)-A(t)\} T\left(t_{1} ; A\left(\tau_{1}\right)\right) \cdots T\left(t_{n} ; A\left(\tau_{n}\right)\right) f\right\| \\
& =\left\|T\left(t_{1} ; A\left(\tau_{1}\right)\right) \cdots T\left(t_{n} ; A\left(\tau_{n}\right)\right)\{A(t+h) f-A(t) f\}\right\| \quad \text { by }(5.1) \\
& \leqq\|A(t+h) f-A(t) f\|<\varepsilon .
\end{aligned}
$$

Also,

$$
M(T, f, \eta, J) \leqq \max _{t \in[0, T]}\|A(t) f\|<\infty .
$$

Hence (A2) holds, with $\delta$ independent of $\eta$ and $J$. 
Some lemmas will be required for the proof of Theorem 1.2. These are generalizations of Lemmas 3.2, 3.3.

Lemma 5.2. Let $T_{1}, \ldots, T_{n}, S_{1}, \ldots, S_{n}$ be contractions in $\mathscr{B}(\mathfrak{X})$. Then for each $f \in \mathfrak{X}$,

$$
\left\|\left(T_{1} \cdots T_{n}\right) f-\left(S_{1} \cdots S_{n}\right) f\right\| \leqq\left\|T_{n} f-S_{n} f\right\|+\sum_{i=1}^{n-1}\left\|\left(T_{i}-S_{i}\right) S_{i+1} \cdots S_{n} f\right\| .
$$

Proof. The proof is by the telescoping method. Let $U_{1}=V_{n}=I$,

$$
U_{i}=T_{1} \cdots T_{i-1}, \quad V_{i}=S_{i+1} \cdots S_{n}, \quad i=1, \ldots, n .
$$

Then

hence

$$
\left(T_{1} \cdots T_{n}\right)-\left(S_{1} \cdots S_{n}\right)=\sum_{i=1}^{n}\left(U_{i} T_{i} V_{i}-U_{i} S_{i} V_{i}\right)
$$

$$
\left\|\left(T_{1} \cdots T_{n}\right) f-\left(S_{1} \cdots S_{n}\right) f\right\| \leqq \sum_{i=1}^{n}\left\|\left(T_{i}-S_{i}\right) V_{i} f\right\|,
$$

and the proof is complete.

Lemma 5.3. Let $A, B \in \mathscr{A}$. Let $f \in \mathscr{D}(A) \cap \mathscr{D}(B)$, and suppose that $T(\cdot ; B) f$ : $R^{+} \rightarrow \mathscr{D}(A)$. Then

$$
\|T(t ; A) f-T(t ; B) f\| \leqq(U) \int_{0}^{t}\|(A-B) T(u ; B) f\| d u
$$

where the integral is an upper Riemann integral (infimum of upper sums).

Proof. For $t \in R^{+}$,

$$
\begin{aligned}
&\|T(t ; A) f-T(t ; B) f\|=\left\|\left(T\left(\frac{t}{n} ; A\right)\right)^{n} f-\left(T\left(\frac{t}{n} ; B\right)\right)^{n} f\right\| \\
& \leqq \sum_{i=1}^{n}\|\{T(t / n ; A)-T(t / n ; B)\} T(t(n-i) / n ; B) f\| \\
&=(t / n) \sum_{i=1}^{n} \|\left\{(t / n)^{-1}(T(t / n ; A)-I)-(t / n)^{-1}\right. \\
&\quad \cdot(T(t / n ; B)-I)\} T(t(n-i) / n ; B) f \| .
\end{aligned}
$$

As $n \rightarrow \infty$, the limit superior of this expression is bounded by the upper integral $(U) \int_{o}^{t}\|(A-B) T(u ; B) f\| d u$. Since the left side of the above inequality does not depend on $n$, the lemma follows.

Proof of Theorem 1.2. Let the hypotheses of the theorem hold. Let $\Delta^{1}, \Delta^{2}$ be two partitions of $[a, b] \subset R^{+}$; for $i=1,2$,

$$
\begin{gathered}
\Delta^{i}: a=t_{0}^{i}<t_{1}^{i}<\cdots<t_{n_{i}}^{i}=b, \quad \tau_{j}^{i} \in\left[t_{j-1}^{i}, t_{j}^{i}\right], \\
\Delta_{j}^{i} t=t_{j}^{i}-t_{j-1}^{i}, \quad\left|\Delta^{i}\right|=\max _{1 \leqq j \leqq n_{i}} \Delta_{j}^{i} t .
\end{gathered}
$$

Let

$$
R_{\Delta^{i}}=T\left(\Delta_{n_{1}}^{i} t ; A\left(\tau_{n_{1}}^{i}\right)\right) \cdots T\left(\Delta_{1}^{i} t ; A\left(\tau_{1}^{i}\right)\right)
$$


First it will be shown that

$$
U(b, a) f=\lim _{|\Delta| \rightarrow 0} R_{\Delta} f
$$

exists for each $f \in \mathfrak{X}$. Since $\left\|R_{\Delta}\right\| \leqq 1$ and $\mathscr{D}$ is dense in $\mathfrak{X}$, it suffices to show that $\lim _{|\Delta| \rightarrow 0} R_{\Delta} f$ exists for each $f \in \mathscr{D}$. To that end, let $\varepsilon>0$ and $f \in \mathscr{D}$ be given. It suffices to find a $\delta>0$ such that

$$
\left\|R_{\Delta^{1}} f-R_{\Delta^{2}} f\right\|<\varepsilon
$$

if $\left|\Delta^{1}\right|,\left|\Delta^{2}\right|<\delta$. As in the proof of Theorem 3.1, let $\Delta^{3}$ be the common refinement of $\Delta^{1}, \Delta^{2}$ :

$$
\Delta^{3}: a=t_{0}^{3}<t_{1}^{3}<\cdots<t_{m}^{3}=b
$$

once $t_{j}^{3}$ has been defined, $t_{j+1}^{3}$ is the smallest of $\left\{t_{1}^{1}, \ldots, t_{n_{1}}^{1}, t_{1}^{2}, \ldots, t_{n_{2}}^{2}\right\}$ which is greater than $t_{j}^{3}$. For $i=1,2, s_{j}^{i}=\tau_{k}^{i}$, where $k$ is the unique subscript such that $t_{k-1}^{i} \leqq t_{j-1}^{3}<t_{j}^{3} \leqq t_{k}^{i}$. Then

$$
R_{\Delta^{i}} f=T\left(\Delta_{m}^{3} t ; A\left(s_{m}^{i}\right)\right) \cdots T\left(\Delta_{1}^{3} t ; A\left(s_{1}^{i}\right)\right) f .
$$

Therefore,

$$
\begin{aligned}
\left\|R_{\Delta^{1}} f-R_{\Delta^{2}} f\right\| & =\| T\left(\Delta_{m}^{3} t ; A\left(s_{m}^{1}\right)\right) \cdots T\left(\Delta_{1}^{3} ; A\left(s_{1}^{1}\right)\right) f \\
& -T\left(\Delta_{m}^{3} t ; A\left(s_{m}^{2}\right)\right) \cdots T\left(\Delta_{1}^{3} t ; A\left(s_{1}^{2}\right)\right) f \| \\
\leqq & \left\|f_{0}\right\|+\sum_{i=0}^{m-2}\left\|\left\{T\left(\Delta_{m-i}^{3} t ; A\left(s_{m-i}^{1}\right)\right)-T\left(\Delta_{m-i}^{3} t ; A\left(s_{m-i}^{2}\right)\right)\right\} g_{m-i}\right\|
\end{aligned}
$$

by Lemma 5.2 , where

$$
f_{0}=T\left(\Delta_{1}^{3} t ; A\left(s_{1}^{1}\right)\right) f-T\left(\Delta_{1}^{3} t ; A\left(s_{1}^{2}\right)\right) f
$$

and

$$
g_{k}=T\left(\Delta_{k-1}^{3} t ; A\left(s_{k-1}^{2}\right)\right) \cdots T\left(\Delta_{1}^{3} t ; A\left(s_{1}^{2}\right)\right) f .
$$

Let $b \leqq T<\infty, \eta=T, J=[0, T]$. Then $g_{k} \in C(f, \eta, J)$. (Actually, $\eta=b-a$. and $J=[a, b]$ will do.) Since $\lim _{\left|\Delta^{1}\right|,\left|\Delta^{2}\right| \rightarrow 0}\left\|f_{0}\right\|=0$, there is a $\delta_{1}>0$ such that $\left\|f_{0}\right\|<\varepsilon / 2$ if $\left|\Delta^{1}\right|,\left|\Delta^{2}\right|<\delta$. Now, by Lemma 5.3,

$$
\begin{aligned}
\|\left\{T\left(\Delta_{m-i}^{3} t ; A\left(s_{m-i}^{1}\right)\right)\right. & \left.-T\left(\Delta_{m-i}^{3} t ; A\left(s_{m-i}^{2}\right)\right)\right\} g_{m-i} \| \\
& \leqq(U) \int_{0}^{\Delta_{m-1}^{3} t}\left\|\left\{A\left(s_{m-i}^{1}\right)-A\left(s_{m-i}^{2}\right)\right\} T\left(u ; A\left(s_{m-i}^{2}\right)\right) g_{m-i}\right\| d u .
\end{aligned}
$$

But also $T\left(u ; A\left(s_{m-i}^{2}\right)\right) g_{m-i} \in C(f, \eta, J)$ with the same $\eta$ and $J$ as above, hence by hypothesis (A2), there is a $\delta_{2}>0$ such that

$$
\left\|\left\{A\left(s_{m-i}^{1}\right)-A\left(s_{m-1}^{2}\right)\right\} T\left(u ; A\left(s_{m-i}^{2}\right)\right) g_{m-i}\right\|<\varepsilon / 2(b-a)
$$


independent of $m, i, u \in\left[0, \Delta_{m-i}^{3} t\right]$, as long as $\left|\Delta^{1}\right|,\left|\Delta^{2}\right|<\delta_{2}$. If $\delta$ is the smaller of $\delta_{1}, \delta_{2}$, then $\left|\Delta^{1}\right|,\left|\Delta^{2}\right|<\delta$ implies

$$
\begin{array}{rlrl}
\left\|R_{\Delta^{1}} f-R_{\Delta^{2}} f\right\| & \leqq\left\|f_{0}\right\|+\sum_{i=0}^{m-2}(U) \int_{0}^{\Delta_{m-i}^{3} t}\left\|\left\{A\left(s_{m-i}^{1}\right)-A\left(s_{m-i}^{2}\right)\right\} T\left(u ; A\left(s_{m-i}^{2}\right)\right) g_{m-i}\right\| d u \\
& <\frac{\varepsilon}{2}+\sum_{i=0}^{m-2}\left(\Delta_{m-i}^{3} t\right) \frac{\varepsilon}{2(b-a)} & \text { by (5.2) and (5.3) } \\
& =\frac{\varepsilon}{2}+\frac{\varepsilon}{2}=\varepsilon .
\end{array}
$$

Hence $U(b, a)=\lim _{|\Delta| \rightarrow 0} R_{\Delta}$ exists in the strong operator topology for all $(b, a) \in D$. Summarizing, given $\varepsilon>0, T>0$, and $f \in \mathscr{D}$, there is a $\delta=\delta(\varepsilon, T, f)>0$ such that

$$
\left\|U(b, a) f-R_{\Delta} f\right\|<\varepsilon
$$

whenever $0 \leqq a \leqq b \leqq T$ and $\Delta$ is any partition of $[a, b]$ such that $|\Delta|<\delta$.

According to Proposition 2.3, $U(\cdot, \cdot)$ is a generalized semigroup in $\mathscr{B}(\mathfrak{X})$. According to Propositions 2.1 and 2.3 , to show that $U(\cdot, \cdot)$ is strongly continuous, it suffices to show that

$$
\lim _{(\tau, \sigma) \rightarrow(t, s) ;(\tau, \sigma) \in D, \tau \leqq t} U(\tau, \sigma) f=U(t, s) f
$$

whenever $(t, s) \in D, f \in \mathfrak{X}$. It suffices to prove this for $f \in \mathscr{D}$, since $\mathscr{D}$ is dense in $\mathfrak{X}$ and $\|U(t, s)\| \leqq 1$.

First consider the case when $s=t$. Then $\sigma \leqq \tau \leqq s=t$. For $f \in \mathscr{D}$,

$$
\begin{aligned}
\|U(\tau, \sigma) f-f\|= & \|U(\tau, \sigma) f-T(\tau-\sigma ; A(\sigma)) f\| \\
& +\|T(\tau-\sigma ; A(\sigma)) f-T(\tau-\sigma ; A(t)) f\|+\|T(\tau-\sigma ; A(t)) f-f\| \\
= & J_{1}+J_{2}+J_{3} .
\end{aligned}
$$

Clearly $J_{3} \rightarrow 0$ as $(\tau, \sigma) \rightarrow(t, t)$. Also $J_{1} \rightarrow 0$ as $(\tau, \sigma) \rightarrow(t, t)$ by (5.5), taking $R_{\Delta}=T(\tau-\sigma ; A(\sigma))$; this corresponds to a trivial partition of $[\sigma, \tau]$. Finally

$$
\begin{aligned}
J_{2} & \leqq(U) \int_{0}^{\tau-\sigma}\|(A(\sigma)-A(t)) T(u ; A(t)) f\| d u \quad \text { by Lemma } 5.3 \\
& \leqq(U) \int_{0}^{\tau-\sigma}\|A(\sigma) T(u ; A(t)) f\| d u+\int_{0}^{\tau-\sigma}\|T(u ; A(t)) A(t) f\| d u \\
& \leqq(\tau-\sigma) M(t, f, t,[0, t])+(\tau-\sigma)\|A(t) f\| \rightarrow 0
\end{aligned}
$$

as $(\tau, \sigma) \rightarrow(t, t)$ by (A2), since $T(u ; A(t)) f \in C(f, t,[0, t])$.

Now let $t>s \geqq 0(\tau \leqq t)$. Let $f \in \mathscr{D}$.

where

$$
U(\tau, \sigma) f-U(t, s) f=\lim _{n \rightarrow \infty}\left(U_{n} f-V_{n} f\right),
$$

$$
\begin{aligned}
& U_{n} f=T\left(\frac{\tau-\sigma}{n} ; A(\tau)\right) T\left(\frac{\tau-\sigma}{n} ; A\left(\sigma+\frac{n-1}{n}(\tau-\sigma)\right)\right) \cdots T\left(\frac{\tau-\sigma}{n} ; A\left(\sigma+\frac{1}{n}(\tau-\sigma)\right)\right) f \\
& V_{n} f=T\left(\frac{t-s}{n} ; A(t)\right) \cdots T\left(\frac{t-s}{n} ; A\left(s+\frac{1}{n}(t-s)\right)\right) f
\end{aligned}
$$


In fact, according to (5.5), given $\varepsilon_{1}>0$ there is a $\delta_{1}>0$ such that $t / n<\delta_{1}$ implies

$$
\left\|U_{n} f-U(\tau, \sigma) f\right\|<\varepsilon_{1}, \quad\left\|V_{n} f-U(t, s) f\right\|<\varepsilon_{1} .
$$

Therefore, to prove that

$$
\lim _{(\tau, \sigma) \rightarrow(t, s)} U(\tau, \sigma) f=U(t, s) f
$$

it suffices to prove the following: given $\varepsilon>0$ and a positive integer $n$,

$$
\limsup _{(\tau, \sigma) \rightarrow(t, s)}\left\|U_{n} f-V_{n} f\right\| \leqq \varepsilon .
$$

Let $\varepsilon>0$ be given, and let $n$ be fixed but arbitrary.

$$
\begin{aligned}
&\left\|U_{n} f-V_{n} f\right\|= \| T\left(\frac{\tau-\sigma}{n} ; A(\tau)\right) \cdots T\left(\frac{\tau-\sigma}{n} ; A\left(\sigma+\frac{1}{n}(\tau-\sigma)\right)\right) f-T\left(\frac{t-s}{n} ; A(t)\right) \\
& \cdots T\left(\frac{t-s}{n} ; A\left(s+\frac{1}{n}(t-s)\right)\right) f \| \\
& \leqq \\
& \sum_{i=1}^{n}\left\|\left\{T\left(\frac{\tau-\sigma}{n} ; A\left(\sigma+\frac{i}{n}(\tau-\sigma)\right)\right)-T\left(\frac{t-s}{n} ; A\left(s+\frac{i}{n}(t-s)\right)\right)\right\} h_{i}\right\| \\
&=\sum_{i=1}^{n} H_{i}
\end{aligned}
$$

by Lemma 5.2, where each $h_{i}$ is an element of $C(f, t,[0, t])$.

$$
\begin{aligned}
H_{i} \leqq & \left\|T\left(\frac{\tau-\sigma}{n} ; A\left(\sigma+\frac{i}{n}(\tau-\sigma)\right)\right) h_{i}-T\left(\frac{\tau-\sigma}{n} ; A\left(s+\frac{i}{n}(t-s)\right)\right) h_{i}\right\| \\
& +\left\|T\left(\frac{\tau-\sigma}{n} ; A\left(s+\frac{i}{n}(t-s)\right)\right) h_{i}-T\left(\frac{t-s}{n} ; A\left(s+\frac{i}{n}(t-s)\right)\right) h_{i}\right\| \\
= & K_{i}^{1}+K_{i}^{2} .
\end{aligned}
$$

The semigroups $\{T(u ; A(v))\}$ are strongly continuous, therefore there is a $\delta_{i}=\delta_{i}(\varepsilon, n)$ such that $K_{i}^{2}<\varepsilon / 2 n$ if $|s-\sigma|+|t-\tau|<\delta_{i}, i=1, \ldots, n$.

Let $k_{i}(u)=T(u ; A(s+i(t-s) / n)) h_{i} ; k_{i}(u) \in C(f, t,[0, t])$ if $0 \leqq u \leqq t$. By Lemma 5.3 and the definition of $k_{i}(u)$,

$$
K_{i}^{1} \leqq(U) \int_{0}^{(\tau-\sigma) / n}\left\|\left\{A\left(\sigma+\frac{i}{n}(\tau-\sigma)\right)-A\left(s+\frac{i}{n}(t-s)\right)\right\} k_{i}(u)\right\| d u .
$$

Since $A(\cdot) g$ is uniformly strongly continuous on the compact interval $[0, t]$, the continuity in addition being uniform in $g \in C(f, t,[0, t])$ by (A2), there is a $\delta_{0}>0$ such that

$$
\|A(u) g-A(v) g\|<\varepsilon / 2 t
$$


whenever $g \in C(f, t,[0, t])$ and $u, v \in[0, t],|u-v|<\delta_{0}$. If $|s-\sigma|+|t-\tau|<\delta_{0} / 2$, then

$$
\begin{aligned}
\left|\left(\sigma+\frac{i}{n}(\tau-\sigma)\right)-\left(s+\frac{i}{n}(t-s)\right)\right| & =\left|(\sigma-s)+\frac{i}{n}\{(\tau-t)+(s-\sigma)\}\right| \\
& \leqq 2|\sigma-s|+|\tau-t|<2 \cdot \frac{\delta_{0}}{2}=\delta_{0}
\end{aligned}
$$

and so

$$
\left\|\{A(\sigma+(i / n)(\tau-\sigma))-A(s+(i / n)(t-s))\} k_{i}(u)\right\|<\varepsilon / 2 t
$$

Hence

$$
K_{i}^{1} \leqq \int_{0}^{(\tau-\sigma) / n} \frac{\varepsilon}{2 t} d u=\frac{\tau-\sigma}{n} \cdot \frac{\varepsilon}{2 t} \leqq \frac{\varepsilon}{2 n}
$$

since $0 \leqq \sigma \leqq \tau \leqq t$.

Combining the estimates gives

$$
\left\|U_{n} f-V_{n} f\right\| \leqq \sum_{i=1}^{n}\left(K_{j}^{1}+K_{i}^{2}\right)<\sum_{i=1}^{n}\left(\frac{\varepsilon}{2 n}+\frac{\varepsilon}{2 n}\right)=\varepsilon
$$

if $|s-\sigma|+|t-\tau|<\delta=\delta(\varepsilon, n)=\min \left(\delta_{0} / 2, \delta_{1}, \ldots, \delta_{n}\right)$. Thus,

$$
\limsup _{(\tau, \sigma) \rightarrow(t, s)}\left\|U_{n} f-V_{n} f\right\| \leqq \varepsilon,
$$

as was to be shown. So the strong continuity of the generalized semigroup $U(\cdot, \cdot)$ on $D$ is completely proved.

It will be shown that for $f \in \mathscr{D}$,

$$
\begin{array}{ll}
\lim _{h \rightarrow 0+} h^{-1}(U(t+h, t) f-f)=A(t) f & \text { for all } t \in R^{+}, \\
\lim _{h \rightarrow 0+} h^{-1}(U(t, t-h) f-f)=A(t) f & \text { for all } t>0 .
\end{array}
$$

If $\Delta$ is a partition of $[a, b], R_{\Delta}(b, a)$ denotes an approximation of $U(b, a)$ corresponding to $\Delta$. To prove (5.6), let $f \in \mathscr{D}$ and $t \in R^{+}$. Using obvious notation,

$$
U(t+h, t) f=\lim _{|\Delta| \rightarrow 0} R_{\Delta}(t+h, t) f
$$

and

$$
\begin{aligned}
\| R_{\Delta}(t & +h, t) f-T(h ; A(t)) f \| \\
& =\left\|T\left(\Delta_{n} t ; A\left(\tau_{n}\right)\right) \cdots T\left(\Delta_{1} t ; A\left(\tau_{1}\right)\right) f-T\left(\Delta_{n} t ; A(t)\right) \cdots T\left(\Delta_{1} t ; A(t)\right) f\right\| \\
& \leqq \sum_{i=1}^{n}(U) \int_{0}^{\Delta_{i} t}\left\|\left\{A\left(\tau_{i}\right)-A(t)\right\} T(u ; A(t)) T\left(\Delta_{i+1} t ; A(t)\right) \cdots T\left(\Delta_{n} t ; A(t)\right) f\right\| d u
\end{aligned}
$$

by Lemmas $5.2,5.3$

$\leqq \sum_{i=1}^{n} \Delta_{i} t \sup \{\|\{A(\tau)-A(t)\} T(v ; A(t)) f\|: v \in[0, h], \tau \in[t, t+h]\}$. 
But $\sum_{i=1}^{n} \Delta_{i} t=h$, and

$$
\limsup _{h \rightarrow 0+}\{\|\{A(\tau)-A(t)\} T(v ; A(t)) f\|: v \in[0, h], \tau \in[t, t+h]\}=0
$$

by (A2). Therefore

$$
\left\|R_{\Delta}(t+h, t) f-T(h ; A(t)) f\right\| \leqq h \xi(h),
$$

where

$$
\xi(h)=\sup \{\|\{A(\tau)-A(t)\} T(v ; A(t)) f\|: v \in[0, h], \tau \in[t, t+h]\},
$$

and $\lim _{h \rightarrow 0+} \xi(h)=0$. Consequently,

$$
\begin{aligned}
& \limsup _{h \rightarrow 0+}\left\|h^{-1}(U(t+h, t) f-f)-A(t) f\right\| \\
& \leqq \limsup _{h \rightarrow 0+}\left\|h^{-1}(U(t+h, t) f-f)-h^{-1}(T(h ; A(t)) f-f)\right\| \\
& +\limsup _{h \rightarrow 0+}\left\|h^{-1}(T(h ; A(t)) f-f)-A(t) f\right\| \\
& =\limsup _{h \rightarrow 0+}\left\|h^{-1}(U(t+h, t) f-T(h ; A(t)) f)\right\|+0 \\
& =\limsup _{h \rightarrow 0+}\left\|h^{-1}\left(\lim _{|\Delta| \rightarrow 0} R_{\Delta}(t+h, t) f-T(h ; A(t)) f\right)\right\| \quad \text { by (5.8) } \\
& \leqq \limsup _{h \rightarrow 0+}\left|h^{-1} \cdot h \xi(h)\right| \\
& =\limsup _{h \rightarrow 0+} \xi(h)=0 \text {. }
\end{aligned}
$$

Thus formula (5.6) holds.

The verification of (5.7) is similar; if $f \in \mathscr{D}$ and $t>0$,

$$
\begin{aligned}
& \underset{h \rightarrow 0+}{\lim \sup }\left\|h^{-1}(U(t, t-h) f-f)-A(t) f\right\| \\
& \leqq \limsup _{h \rightarrow 0+}\left\|h^{-1}(U(t, t-h) f-f)-h^{-1}(T(h ; A(t)) f-f)\right\| \\
& \quad+\limsup _{h \rightarrow 0+}\left\|h^{-1}(T(h ; A(t)) f-f)-A(t) f\right\| \\
&= \limsup _{h \rightarrow 0+} h^{-1}\|U(t, t-h) f-T(h ; A(t)) f\|+0 \\
&= \limsup _{h \rightarrow 0+} h^{-1} \cdot \sup \{\|\{A(t)-A(\tau)\} T(v ; A(t)) f\|: \tau \in[0, h], \tau \in[t-h, t]\} \\
&= 0 .
\end{aligned}
$$

Now suppose that each $U(b, a)$ leaves $\mathscr{D}$ invariant. For $f \in \mathscr{D}$ and $s \in R^{+}$, if

$$
u(\cdot)=U(\cdot, s) f:[s, \infty) \rightarrow \mathscr{D},
$$

then $u(s)=f,\|u(t)\| \leqq\|u(\tau)\| \leqq\|f\|$ whenever $s \leqq \tau \leqq t<\infty$, and if $t \geqq s$,

$$
\begin{aligned}
\frac{d^{+}}{d t} u(t) & =\lim _{h \rightarrow 0+} h^{-1}(U(t+h, s) f-U(t, s) f) \\
& =\lim _{h \rightarrow 0+} h^{-1}(U(t+h, t)-I) U(t, s) f=A(t) f
\end{aligned}
$$

by (5.6), since $U(t, s) f \in \mathscr{D}$. 
The uniqueness is an immediate consequence of [6, Theorem 1, p. 210]. This completes the proof.

Proof of Lemma 5.1. Let the hypotheses of the lemma hold. Let $f \in \mathscr{D}, \tau \in R^{+}$. It will now be shown that $U(t, s) f \in \mathscr{D}(A(\tau))$. To that end, let $\left\{\Delta_{n}\right\}$ be a sequence of partitions of $[s, t]$ such that $\lim _{n \rightarrow \infty}\left|\Delta_{n}\right|=0$; let $R_{n}=R_{\Delta_{n}}(t, s)$ be corresponding approximations for $U(t, s)$. Since each $T(u ; A(v))$ leaves $\mathscr{D}$ invariant, $R_{n} f \in \mathscr{D}$ $\subset \mathscr{D}(A(\tau))$ for each positive integer $n$. By assumption (A2), $\left\|A(\tau) R_{n} f\right\|$ is a bounded numerical sequence since

$$
\left\|A(\tau) R_{n} f\right\| \leqq \sup \{\|A(\tau) g\|: g \in C(f, t,[0, t])\}<\infty .
$$

Consequently $U(t, s) f \in \mathscr{D}(A(\tau))$ and

according to the following result.

$$
A(\tau) U(t, s) f=\text { weak } \lim _{n \rightarrow \infty} A(\tau) R_{n} f
$$

LEMMA 5.4. Let $\mathfrak{X}$ be reflexive. Let $A$ be a closed densely defined operator on $\mathscr{D}(A) \subset \mathfrak{X}$ to $\mathfrak{X}$. If $\left\{g_{n}\right\}$ is a weakly convergent sequence in $\mathscr{D}(A)$ such that $\left\|A g_{n}\right\|$ is bounded, then $\left\{A g_{n}\right\}$ converges weakly to an element of $\mathscr{D}(A)$ and

$$
A\left(\text { weak } \lim _{n \rightarrow \infty} g_{n}\right)=\text { weak } \lim _{n \rightarrow \infty} A g_{n} \text {. }
$$

For proof see Kato [6, p. 214].

It is of interest to know when $(d / d t) u(\cdot)$ exists as a two-sided derivative, when $u(\cdot)$ is the solution of the Cauchy problem

$$
\left(d^{+} / d t\right) u(t)=A(t) u(t), u(s)=f
$$

in the statement of Theorem 1.2. Sufficient for this is that $A(\cdot) u(\cdot):[s, \infty) \rightarrow \mathfrak{X}$ is strongly continuous. To see this set

$$
v(t)=\int_{s}^{t} A(t) u(t) d t+f
$$

Then $v$ is strongly continously differentiable and $\left(d^{+} / d t\right)(u(t)-v(t)) \equiv 0$. Hence $u(t) \equiv v(t)$ since $v(s)=u(s)$.

The proof of Theorem 1.2 can be modified to yield a new proof of the following classical result.

COROLlARY 5.5. Let $\mathscr{B}$ be a Banach algebra with identity. Let $A(\cdot): R^{+} \rightarrow \mathscr{B}$ be continuous (in the norm topology of $\mathscr{B}$ ). Then $A(\cdot)$ is the infinitesimal propagator of $a$ continuous generalized semigroup in $\mathscr{B}$. Thus the Cauchy problem (1.1) is well posed.

The details are omitted.

6. Another example. In this section a general example will be given for which the hypotheses of Theorem 1.2 are satisfied, but for which the conditions of Theorem 1.1 fail to hold.

$$
\frac{\partial u}{\partial t}=a(t, x) \frac{\partial u}{\partial x}+b(t, x) u \quad u(0, x)=f(x)
$$


Let $\mathfrak{X}$ be $C[-\infty, \infty]$ with the supremum norm or $L^{p}(-\infty, \infty), 1 \leqq p<\infty$. Suppose that the following conditions hold:

(C1) For each $t \in R^{+}, a(t, \cdot), b(t, \cdot), a_{2}(t, \cdot), b_{2}(t, \cdot) \in \mathfrak{X}$ (here $a_{2}(t, x)=$ $(\partial / \partial x) a(t, x))$.

(C2) The maps $t \rightarrow a(t, \cdot), t \rightarrow b(t, \cdot)$ are strongly continuous on $R^{+}$to $\mathfrak{X}$.

(C3) $\sup _{t \in[0, \tau]}\left\|a_{2}(t, \cdot)\right\|<\infty, \sup _{t \in[0, \tau]}\left\|b_{2}(t, \cdot)\right\|<\infty$ for each $\tau \in R^{+}$.

(C4) $f \in \mathscr{D}=\left\{h: h, h^{\prime} \in \mathfrak{X}\right\}$.

(C5) $b(t, x) \leqq 0$ for all $t \in R^{+}, x \in R^{1}$.

Under these conditions the hypotheses of Theorem 1.2 are satisfied. The proof will be given for $\mathfrak{X}=C[-\infty, \infty]$. As was shown in $\S 4$, Assumption (C5) can be made without any loss of generality. A preliminary result is given first.

LEMMA 6.1. Let $a, b, f$ be continuous functions on $R^{1}$, and suppose that a has $a$ continuous derivative. Let $\gamma(\cdot, x)$ be the unique solution of the ordinary differential equation

$$
d y / d t=a(y), \quad y(0)=x .
$$

Then $u$ given by

$$
u(t, x)=\exp \left\{\int_{0}^{t} b(\gamma(s, x)) d s\right\} f(\gamma(t, x))
$$

satisfies

$$
\frac{\partial u}{\partial t}=a(x) \frac{\partial u}{\partial x}+b(x) u \quad u(0, x)=f(x)
$$

for all $t \in R^{+}, x \in R^{1}$.

Proof. This result is easily proved by differentiating $u$ given by (6.2).

Since it is not obvious that formula (6.2) gives the solution to problem (6.3), a formal derivation of (6.2) will now be given, using a result of semigroup theory.

A theorem of Trotter [12] (see also Nelson [11]) states that if $\left\{T(t), t \in R^{+}\right\}$, $\left\{S(t), t \in R^{+}\right\},\left\{U(t), t \in R^{+}\right\}$are semigroups with infinitesimal generators $A, B$, $A+B$ respectively, then

$$
U(t)=\lim _{n \rightarrow \infty}(S(t / n) T(t / n))^{n},
$$

the limit being in the strong operator topology. $A=a(x)(d / d x)$ and $B=b(x) I$ (with properly assigned domains) generate, respectively, semigroups given by the formulas

$$
(T(t) f)(x)=f(\gamma(t, x)), \quad(S(t) f)(x)=e^{t b(x)} f(x) .
$$

$A+B=a(x)(d / d x)+b(x) I$ generates the semigroup $\left\{U(t), t \in R^{+}\right\}$, where

$$
U(t) f=\lim _{n \rightarrow \infty}(S(t / n) T(t / n))^{n},
$$


and $u(t, x)=(U(t) f)(x)$ satisfies (6.3). A simple induction argument shows that

$$
(S(t / n) T(t / n))^{n} f(x)=\exp \left\{\sum_{i=0}^{n-1} b(\gamma(i t / n, x)) \frac{t}{n}\right\} f(\gamma(t, x)) .
$$

Letting $n \rightarrow \infty$, the representation (6.2) is obtained.

Equation (6.1) can be written as

$$
u_{t}=A(t) u, \quad u(0)=f .
$$

A straightforward calculation shows that if (C1)-(C5) hold, then the formula

$$
T(t ; A(\tau)) g(x)=\exp \left\{\int_{0}^{t} b^{\tau}\left(\gamma^{\tau}(\xi, x)\right) d \xi\right\} g\left(\gamma^{\tau}(t, x)\right)
$$

defines a strongly continuous contraction semigroup in $\mathscr{B}(\mathfrak{X})$; here $b^{\tau}(y)=b(\tau, y)$, and $\gamma^{\tau}(\cdot, \cdot)$ is associated with $a^{\tau}(\cdot)=a(\tau, \cdot)$ as in Lemma 6.1. Moreover, $\mathscr{D} \subset \mathscr{D}(A(\tau))$ for each $\tau \in R^{+}$,

$$
A(\tau) g=a(\tau, \cdot) g^{\prime}+b(\tau, \cdot) g
$$

for $\tau \in R^{+}, g \in \mathscr{D}$; and each $T(t ; A(\tau))$ leaves $\mathscr{D}$ invariant.

It remains to show that condition (A2) holds. To that end, let $f \in \mathscr{D}, \eta>0$, and let $J$ be a compact interval in $R^{+}$. Let

$$
g=T\left(t_{1} ; A\left(\tau_{1}\right)\right) \cdots T\left(t_{n} ; A\left(\tau_{n}\right)\right) f \in C(f, \eta, J) .
$$

Recall that $\mathfrak{X}=C[-\infty, \infty]$. Let

$$
k_{m}(x)=\gamma^{\tau_{m}}\left(t_{m}, x\right), \quad m=1, \ldots, n,
$$

and let $p_{0}(x) \equiv 0, p_{1}(x)=k_{1}(x), p_{2}(x)=k_{1}\left(k_{2}(x)\right)$, and in general

$$
p_{m}(x)=k_{1}\left(k_{2}\left(\cdots\left(k_{m}(x)\right) \cdots\right)\right) \text {. }
$$

Note that

$$
\begin{aligned}
& p_{1}^{\prime}(x)=k_{1}^{\prime}(x) \\
& p_{2}^{\prime}(x)=k_{1}^{\prime}\left(k_{2}(x)\right) k_{2}^{\prime}(x), \ldots, \\
& p_{m}^{\prime}(x)=k_{1}^{\prime}\left(k_{2}\left(\cdots\left(k_{m}(x)\right) \cdots\right)\right) \cdots k_{m-1}^{\prime}\left(k_{m}(x)\right) k_{m}^{\prime}(x) .
\end{aligned}
$$

According to a known result from the theory of ordinary differential equations [1, pp. 22-25],

$$
k_{1}^{\prime}(x)=\frac{\partial}{\partial x} \gamma^{\tau_{i}}\left(t_{i}, x\right)=\exp \left\{\int_{0}^{t_{i}} a_{2}\left(s, \gamma^{\tau_{i}}(s, x)\right) d s\right\}
$$

Hence

independent of $x$, where

$$
0 \leqq k_{i}^{\prime}(x) \leqq e^{t_{i} N\left(\tau_{i}\right)}
$$

$$
N(\tau)=\sup _{y \in R^{1}} \max \left(\left|a_{2}(y, \tau)\right|,\left|b_{2}(y, \tau)\right|\right) .
$$


Set $N(J)=\sup \{N(\tau): \tau \in J\}$; then $N(J)<\infty$ by (C3). It follows that

$$
0 \leqq p_{m}^{\prime}(x) \leqq \prod_{i=1}^{m} \exp \left(t_{i} N\left(\tau_{i}\right)\right) \leqq \exp \left\{N(J) \sum_{i=1}^{m} t_{i}\right\} \leqq \exp (N(J) \eta)
$$

Set

$$
e_{i}(x)=\exp \left\{\int_{0}^{t_{i}} b^{\tau_{i}}\left(\gamma^{\tau_{i}}\left(u, p_{i-1}(x)\right)\right) d u\right\}, \quad i=1, \ldots, n .
$$

By induction,

$$
g(x)=\prod_{i=1}^{n} e_{i}(x) f\left(p_{n}(x)\right)
$$

Therefore,

$$
\begin{aligned}
g^{\prime}(x)= & \prod_{i=1}^{n} e_{i}(x) f^{\prime}\left(p_{n}(x)\right) p_{n}^{\prime}(x) \\
& +\sum_{j=1}^{n} \prod_{i \neq j} e_{i}(x) f\left(p_{n}(x)\right) p_{j-1}^{\prime}(x) \cdot \int_{0}^{t_{j}} b^{\tau_{j}^{\prime}}\left(\gamma^{\tau_{j}}\left(u, p_{j-1}(x)\right)\right) d u,
\end{aligned}
$$

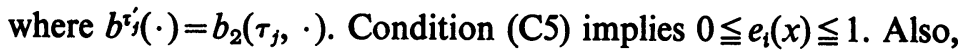

$$
\left|\int_{0}^{t_{j}} b^{\tau_{j}^{\prime}}\left(\gamma^{\tau_{j}}\left(u, p_{j-1}(x)\right)\right) d u\right| \leqq t_{j} N(J) .
$$

Combining all these estimates,

$$
\left|g^{\prime}(x)\right| \leqq\left\|f^{\prime}\right\| \exp (N(J) \eta)+\|f\| \exp (N(J) \eta) \sum_{j=1}^{n} t_{j} N(J) .
$$

Hence

$$
\left\|g^{\prime}\right\| \leqq \exp (N(J) \eta)\left\{\left\|f^{\prime}\right\|+N(J) \eta\|f\|\right\} .
$$

Also, $\|g\| \leqq\|f\| ;$ therefore

$$
\begin{aligned}
\|A(t) g-A(s) g\|= & \sup _{x \in R^{1}}\left|a(t, x) g^{\prime}(x)-a(s, x) g^{\prime}(x)+b(t, x) g(x)-b(s, x) g(x)\right| \\
\leqq & \|a(t, \cdot)-a(s, \cdot)\| \exp (N(J) \eta)\left\{\left\|f^{\prime}\right\|+N(J) \eta\|f\|\right\} \\
& +\|b(t, \cdot)-b(s, \cdot)\|\|f\| .
\end{aligned}
$$

Also,

$$
\begin{aligned}
M(T, f, \eta, J)= & \sup \{\|A(t) g\|: g \in C(f, \eta, J), t \in[0, T]\} \\
\leqq & \max _{t \in[0, T]}\|a(t, \cdot)\| \exp (N(J) \eta)\left\{\left\|f^{\prime}\right\|+N(J) \eta\|f\|\right\} \\
& +\max _{t \in[0, T]}\|b(t, \cdot)\|\|f\|<\infty .
\end{aligned}
$$

Therefore (A2) holds, and so by Theorem 1.2, the generalized semigroup governing equation (6.1) and having $A(\cdot)$ as its infinitesimal propagator exists. 
For $s, t, \sigma, \tau \in R^{+}$,

$$
\begin{aligned}
& T(t ; A(\tau)) T(s ; A(\sigma)) f(x) \\
& \quad=\exp \left\{\int_{0}^{t} b^{\tau}\left(\gamma^{\tau}\left(\xi_{1}, x\right)\right) d \xi_{1}\right\} \exp \left\{\int_{0}^{s} b^{\sigma}\left(\gamma^{\sigma}\left(\xi_{2}, \gamma^{\tau}(t, x)\right)\right) d \xi_{2}\right\} f\left(\gamma^{\sigma}\left(s, \gamma^{\tau}(t, x)\right)\right),
\end{aligned}
$$

and

$$
\begin{aligned}
& T(s ; A(\sigma)) T(t ; A(\tau)) f(x) \\
& \quad=\exp \left\{\int_{0}^{s} b^{\sigma}\left(\gamma^{\sigma}\left(\xi_{1}, x\right)\right) d \xi\right\} \exp \left\{\int_{0}^{t} b^{\tau}\left(\gamma^{\tau}\left(\xi_{2}, \gamma^{\sigma}(s, x)\right)\right) d \xi_{2}\right\} f\left(\gamma^{\tau}\left(t, \gamma^{\sigma}(s, x)\right)\right) .
\end{aligned}
$$

Only in special cases will

$$
T(t ; A(\tau)) T(s ; A(\sigma))=T(s ; A(\sigma)) T(t ; A(\tau)) .
$$

In fact, roughly speaking, (6.4) will hold for all $s, t, \sigma, \tau$ (i.e. condition (B1) of $\S 3$ will hold) if and only if $a, b$ are of the form

$$
a(t, x)=\alpha(t) h(x), \quad b(t, x)=\alpha(t) k(x)+\beta(t) .
$$

7. Concluding remarks. The first fundamental contributions to nonstationary abstract evolution equations using semigroup theory were made by Kato [6], [7]. His basic assumptions were smoothness conditions on the operator valued function $B(s, t)=(I-A(t))(I-A(s))^{-1}$. Also, in [6], $\mathscr{D}(A(t))$ was assumed to be independent of $t$, but a less restrictive assumption was made in [7].

Our smoothness conditions and Kato's smoothness conditions are of a different nature, and it is difficult to compare them. Nevertheless, a few remarks are in order.

It is of interest to have the domain of $A(t)$ vary quite generally with $t$. Sometimes $A(t)$ arises as the closure of a differential operator, and $\mathscr{D}(A(t))$ is not explicitly known (see $\$ 4$ ). Condition (B2)' of $\$ 3$ seems to be a satisfactory condition in that $\mathscr{D}(A(t))$ can vary with $t$, and the smoothness requirement on $A(\cdot)$ is easy to verify in practice. On the other hand, the commutativity. hypothesis seriously restricts the applicability of the results to concrete problems. However, in case (B1) holds, then, as exemplified in $\S 4$, satisfactory results can be obtained.

It is, as indicated above, difficult to compare condition (A2) with Kato's conditions. Also, Kato's methods of proof in [6], [7] are different from the methods employed here. There are problems to which the results of this paper can be applied, but to which Kato's results do not apply (cf. §4); on the other hand, there exist examples to which Kato's theorems apply, but to which the results presented here do not seem to be applicable.

\section{REFERENCES}

1. E. Coddington and N. Levinson, Theory of ordinary differential equations, McGraw-Hill, New York, 1955.

2. N. Dunford and J. T. Schwartz, Linear operators, Part I: General theory, Interscience, New York, 1958. 
3. W. Feller, An introduction to probability theory and its applications, Vol. II, Wiley, New York, 1966.

4. A. Friedman, Partial differential equations of parabolic type, Prentice-Hall, Englewood Cliffs, N. J., 1964.

5. E. Hille and R. S. Phillips, Functional analysis and semi-groups, Amer. Math. Soc. Colloq. Publ., Vol. 31, Amer. Math. Soc., Providence, R. I., 1957.

6. T. Kato, Integration of the equation of evolution in a Banach space, J. Math. Soc. Japan 5 (1953), 208-234.

7. - On linear differential equations in Banach spaces, Comm. Pure Appl. Math. 9 (1956), 479-486.

8. - Perturbation theory for linear operators, Springer, New York, 1966.

9. P. D. Lax and A. N. Milgram, Parabolic equations, Ann. of Math. Studies, No. 33, Princeton Univ. Press, Princeton, N. J., 1954, pp. 167-190.

10. E. Nelson, An existence theorem for second order parabolic equations, Trans. Amer. Math. Soc. 88 (1958), 414-429.

11. - - Feynman integrals and the Schrödinger equation, J. Math. Phys. 5 (1964), 332-343.

12. H. F. Trotter, On the product of semi-groups of operators, Proc. Amer. Math. Soc. 10 (1959), 545-551.

13. K. Yosida, Functional analysis, Springer, Berlin, 1965.

Carnegie Inistitute of Technology, Pittsburgh, Pennsylvania

InSTITUTE FOR ADVANCEd STUDY,

Princeton, New Jersey 